\title{
Transgenic Archaerhodopsin-3 Expression in Hypocretin/Orexin Neurons Engenders Cellular Dysfunction and Features of Type 2 Narcolepsy
}

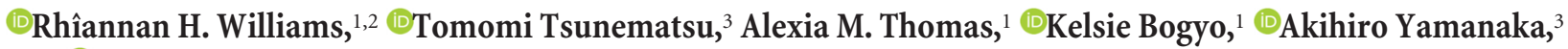 \\ and ${ }^{-T h o m a s ~ S . ~ K i l d u f f ~}{ }^{1}$ \\ ${ }^{1}$ Center for Neuroscience, Biosciences Division, SRI International, Menlo Park, California 94025, ${ }^{2}$ Institute for Neurogenomics, Helmholtz Zentrum \\ München, German Research Centre for Environmental Health, Neuherberg 85764, Germany, and ${ }^{3}$ Research Institute of Environmental Medicine, Nagoya \\ University, Nagoya, 464-8601, Japan
}

Narcolepsy, characterized by excessive daytime sleepiness, is associated with dysfunction of the hypothalamic hypocretin/orexin (Hcrt) system, either due to extensive loss of Hcrt cells (Type 1, NT1) or hypothesized Hcrt signaling impairment (Type 2, NT2). Accordingly, efforts to recapitulate narcolepsy-like symptoms in mice have involved ablating these cells or interrupting Hcrt signaling. Here, we describe orexin/Arch mice, in which a modified archaerhodopsin-3 gene was inserted downstream of the prepro-orexin promoter, resulting in expression of the yellow light-sensitive Arch-3 proton pump specifically within Hcrt neurons. Histological examination along with ex vivo and in vivo electrophysiological recordings of male and female orexin/Arch mice demonstrated silencing of Hcrt neurons when these cells were photoilluminated. However, high expression of the Arch transgene affected cellular and physiological parameters independent of photoillumination. The excitability of Hcrt neurons was reduced, and both circadian and metabolic parameters were perturbed in a subset of orexin/Arch mice that exhibited high levels of Arch expression. Orexin/Arch mice also had increased REM sleep under baseline conditions but did not exhibit cataplexy, a sudden loss of muscle tone during wakefulness characteristic of NT1. These aberrations resembled some aspects of mouse models with Hcrt neuron ablation, yet the number of Hcrt neurons in orexin/Arch mice was not reduced. Thus, orexin/Arch mice may be useful to investigate Hcrt system dysfunction when these neurons are intact, as is thought to occur in narcolepsy without cataplexy (NT2). These results also demonstrate the utility of extended phenotypic screening of transgenic models when specific neural circuits have been manipulated.

Key words: hypothalamus; narcolepsy; NREM sleep; optogenetic; REM sleep; wakefulness

Significance Statement

Optogenetics has become an invaluable tool for functional dissection of neural circuitry. While opsin expression is often achieved by viral injection, stably integrated transgenes offer some practical advantages. Here, we demonstrate successful transgenic expression of an inhibitory opsin in hypocretin/orexin neurons, which are thought to promote or maintain wakefulness. Both brief and prolonged illumination resulted in inhibition of these neurons and induced sleep. However, even in the absence of illumination, these cells exhibited altered electrical characteristics, particularly when transgene expression was high. These aberrant properties affected metabolism and sleep, resulting in a phenotype reminiscent of the narcolepsy Type 2, a sleep disorder for which no good animal model currently exists.

\section{Introduction}

The hypocretin/orexin (Hcrt) system is a hypothalamic neuropeptide system that is involved in behavioral arousal, metabo- lism, reward, stress, and neuroendocrine function (Sakurai, 2007; Mahler et al., 2014; Li et al., 2017). Defects in this system, either presynaptically or postsynaptically, result in Type 1 narcolepsy 
(NT1) in humans and animals (Scammell, 2015; Black et al., 2017a; Bassetti et al., 2019; Szabo et al., 2019). Since the primary symptoms of NT1 are excessive daytime sleepiness (EDS) and cataplexy, the Hcrt system is widely acknowledged to promote wakefulness and maintain muscle tone. The Hcrt system also has a role in energy metabolism and homeostasis (Hara et al., 2001; Yamanaka et al., 2003b; Funato et al., 2009). In support of this concept, the phenotype of narcoleptic mice (Hara et al., 2001; Inutsuka et al., 2014) replicates the increased body mass index reported in narcoleptic patients (Schuld et al., 2000, 2002).

Although afferent control of Hcrt neurons is relatively well characterized (Sakurai et al., 2005; Yoshida et al., 2006; Sakurai, 2007), and the efferent projections of the Hcrt neurons have been known for some time (Peyron et al., 1998; Date et al., 1999), relatively little progress in understanding the functionality of Hcrt efferents was made until the advent of optogenetics. An optogenetic study of the Hcrt system was the first in vivo implementation of this technology (Adamantidis et al., 2007), and subsequent optogenetic studies have also "shed light" on Hcrt function. For example, the short latency awakenings from sleepevoked by channelrhodopsin-2 (ChR2)-mediated Hcrt neuron photoactivation (Adamantidis et al., 2007) have been shown to be sensitive to the sleep pressure induced by sleep deprivation (SD) (Carter et al., 2009). Photoinhibition of locus ceruleus (LC) neurons blocks these Hcrt-mediated sleep-to-wake transitions (Carter et al., 2012), implying that an Hcrt $\rightarrow$ LC pathway may be one circuit underlying the cortical activation that occurs during wakefulness.

In contrast to neural activation by ChR2, optogenetic neuronal silencing has been achieved by expression of the orange lightsensitive protein halorhodopsin, which opens a chloride channel (Han and Boyden, 2007; Zhang et al., 2007), and the yellow lightsensitive proteins archaerhodopsin-3 (Arch) or ArchT, both of which activate a proton pump (Chow et al., 2010; Han et al., 2011). Using these tools, photoinhibition of Hcrt neurons with halorhodopsin (Tsunematsu et al., 2011) or ArchT (Tsunematsu et al., 2013) has been shown to increase time spent in slow-wave sleep during the active phase. In vitro optogenetics combined with pharmacology has also revealed the relative contributions of glutamate- versus Hcrt-mediated excitation of the tuberomammillary neurons (Schöne et al., 2012) and provided evidence for corelease of these neurotransmitters onto Hcrt cells (Schöne et al., 2014). Similar approaches have demonstrated that Hcrt neurons can inhibit melanin-concentrating hormone cells (ApergisSchoute et al., 2015).

In the present study, we characterize a new transgenic mouse line, orexin/Arch mice, to further understand the Hcrt/orexin system. During the course of in vitro electrophysiological experiments, a surprising degree of variability in the response of orexin/ Arch-expressing cells to stimulation was evident. Further analysis revealed that high levels of Arch transgene expression negatively affected Hcrt neuronal excitability in the absence of any illumination and significantly altered body weight and REM sleep. Thus, orexin/Arch mice may be a useful tool for study of energy metabolism as well as sleep-wake control, and these data underscore the need for detailed phenotypic screening of transgenic mouse models when specific neural circuits are targeted.

\section{Materials and Methods}

\section{Animals}

All experimental procedures conducted at SRI International were approved by the Institutional Animal Care and Use Committee at SRI International and were conducted in accordance with the principles set forth in the Guide for Care and Use of Laboratory Animals, National Research Council. All experimental procedures conducted in Japan involving animals were approved by the National Institute for Physiological Sciences Animal Care and Use Committee and were in accordance with National Institutes of Health guidelines. All efforts were made to minimize animal suffering or discomfort and to reduce the number of animals used. All rodents were maintained under $12 \mathrm{~h}$ light/12 h dark (LD) conditions at $22 \pm 2^{\circ} \mathrm{C}$ and $50 \pm 25 \%$ relative humidity with food and water ad libitum.

\section{Generation of orexin/Arch mice}

The transgenic construct was made by substituting the $n L a c Z$ gene (SalIBamHI fragment) of the orexin $/ n L a c Z$ construct with the $1.5 \mathrm{~kb}$ Arch fragment (Han and Boyden, 2007; Chow et al., 2010), which is expressed as a fusion protein with eGFP. The transgene was excised and microinjected into pronuclei of fertilized mouse eggs (BDF1 mice) to generate transgenic founders. Founders were bred with BDF1 mice to produce stable orexin/Arch transgenic lines. A total of 12 transgene-positive founders were obtained. Analysis of the N1 generation demonstrated that only one line (line D3) showed strong expression of Arch, so line D3 was used for subsequent experiments. The mice studied in Japan and at SRI International were derived from same founders and are available from the RIKEN BioResource Center (RBRC05845). At SRI International, orexin/Arch mice were backcrossed with C57BL/6J mice.

\section{Genotyping}

DNA was extracted and isolated from tail snips. Quantitative PCR ( $\mathrm{qPCR}$ ) was used to determine transgene insertion and relative abundance. The $20 \mu \mathrm{l}$ qPCR contained $4 \mu \mathrm{l}$ of target and internal positive control primers, $5 \mu \mathrm{l}$ nuclease-free water, $1 \mu \mathrm{l}$ of DNA sample $(<100$ $\mathrm{ng}$ ), and $10 \mu \mathrm{l}$ of KAPA SYBR FAST qPCR master mix. At SRI International, qPCR analysis used a 7300 Real-Time PCR System (Applied Biosciences) running the following program: $95^{\circ} \mathrm{C}$ for $10 \mathrm{~min}, 42$ cycles of $95^{\circ} \mathrm{C}$ for $15 \mathrm{~s}$, and $60^{\circ} \mathrm{C}$ for $1 \mathrm{~min}$. At Nagoya University, qPCR analysis was conducted using the StepOne Real-Time PCR System (Applied Biosciences) using the following program: $95^{\circ} \mathrm{C}$ for $30 \mathrm{~s}, 40$ cycles at $95^{\circ} \mathrm{C}$ for $3 \mathrm{~s}$, and $60^{\circ} \mathrm{C}$ for $30 \mathrm{~s}$. The ramp of each machine was set to the default. The primers chosen provided the best melting curves. Target primer sequences were as follows: hHCRT1prom1 forward, CCGGGAAAGGA GATGTCTGT, hHCRTprom1 reverse, CGCCACCTCAGAGCACTA TA; internal positive control promoters: mTfrc forward, TGGGCAC TAGATTGGATACCT, mTfrc reverse, GTCCAGGTTCAATTCAAC GTCA (all from Midland Certified Reagent). $C_{t}$ values were assessed relative to an original founder mouse with high Arch transgene expression (aHE). Mice with $\mathrm{qPCR}$ values $\geq 0.9$ were classified as aHE, and those $<0.9$ were classified as low Arch transgene expressers (aLE).

\section{Immunohistochemistry and cell counting}

Tissue preparation. Male and female orexin/Arch transgenic mice that were killed for histological examination received a terminal injection of SomnaSol (Henry Schein) before transcardial perfusion with PBS containing heparin followed by $4 \%$ PFA. After removal of the brain, the tissue was cryoprotected ( $30 \%$ sucrose, $0.1 \mathrm{M}$ PBS) and cut into 6 series of $30 \mu \mathrm{m}$ thick coronal sections in preparation for immunohistochemical processing.

Immunostaining. For double-fluorescent immunohistochemistry of orexin-containing neurons, guinea pig anti-orexin antiserum (1:800, Vector Laboratories) and AlexaFluor-594-labeled goat anti-guinea pig IgG (1:800, Invitrogen) were used to detect Hcrt/orexin neurons in combination with rabbit anti-GFP antiserum (1:1000, Invitrogen) and AlexaFluor-488-labeled anti-rabbit IgG (1:800, Invitrogen) to detect the Arch-eGFP transgene in Hcrt neurons. For triple-fluorescent immunohistochemistry, goat anti-orexin-A (1:3000, Santa Cruz Biotechnology, catalog \#sc-8070; RRID:AB_653610) and goat anti-orexin-B (1:3000, Santa Cruz Biotechnology, catalog \#sc-8071; RRID:AB_653612) antibodies were processed sequentially to prevent secondary signal crossreactivity; the Arch transgene was then detected with chicken anti-GFP antiserum (1:1000, Abcam, catalog \#ab13970; RRID:AB_300798) and AlexaFluor-488 affiniPure donkey anti-chicken IgG (1:500, Jackson ImmunoResearch Laboratories). The orexin-A antisera was detected with 
AlexaFluor-594 affiniPure donkey anti-goat IgG (1:500, Jackson ImmunoResearch Laboratories), whereas the orexin-B antisera was detected with AMCA-streptavidin (1:500, Jackson ImmunoResearch Laboratories) after incubation with Affinipure donkey anti-goat IgG (1:1000, Jackson ImmunoResearch Laboratories).

Innervation of downstream targets from Hcrt/orexin neurons. To determine whether the Arch transgene was expressed within orexin-containing fibers, we detected Arch-eGFP with chicken anti-GFP antiserum (1:1000, Abcam, catalog \#ab13970; RRID:AB_300798) before secondary incubation of Affinipure donkey anti-chicken IgG (1:1000, Jackson ImmunoResearch Laboratories). Arch-eGFP was detected with nickelenhanced 3,3' diaminobenzidine tetrahydrochloride (nDAB; $30 \mathrm{~s}$; SK4100, Vector Laboratories).

Determination of Hcrt/orexin neuron size. Goat anti-orexin-A (1:3000, Santa Cruz Biotechnology, catalog \#sc-8070; RRID:AB_653610) and goat anti-orexin-B (1:3000, Santa Cruz Biotechnology, catalog \#sc-8071; RRID:AB_653612) antibodies were combined and detected with Affinipure donkey anti-goat IgG (1:1000, Jackson ImmunoResearch Laboratories) and 3,3' diaminobenzidine tetrahydrochloride (DAB; 2 min; SK4100, Vector Laboratories). Sections were mounted on Superfrost slides and coverslipped. Using a CTR 5000 microscope (Leica Microsystems), images of the lateral hypothalamic area (LHA) containing orexinpositive neurons were captured for processing in ImageJ.

Colocalization cell counts. Bilateral counts from 3 to 6 sections were taken from each mouse. All sections were mounted on Superfrost slides, coverslipped, and visualized on a CTR 5000 microscope (Leica Microsystems). Analyses were conducted blind to genotype and condition. Transduction efficiency of the Arch-eGFP transgene within the orexincontaining neurons was calculated by counting the number of Arch$\mathrm{eGFP}^{+}$/orexin-immunoreactive (IR) ${ }^{+}$cells, and the total number of orexin-IR ${ }^{+}$cell bodies and then calculating the percentage of doublelabeled cells relative to the total number of orexin-expressing neurons. The same process was applied to calculate c-FOS activation within the orexin-containing neurons. Arch transduction specificity was calculated as the proportion of GFP-IR ${ }^{+}$cells that coexpressed orexin-IR ${ }^{+}$relative to the total number of GFP-IR ${ }^{+}$cells. The region measured included all orexin-IR ${ }^{+}$cell bodies within the tuberal hypothalamus in the sections used for immunohistochemical quantification (bregma: $-1.30 \mathrm{~mm}$ to $-1.70 \mathrm{~mm}$ ) (Franklin and Paxinos, 2008).

The same analyses were performed to calculate Arch transgene expression within either orexin-A-IR ${ }^{+}$or orexin-B-IR ${ }^{+}$neurons. We found no differences in Arch transgene expression between medial and lateral aspects of the tuberal hypothalamus, nor between orexin-A-IR ${ }^{+}$or orexin$\mathrm{B}-\mathrm{IR}^{+}$neurons. Therefore, we combined orexin antisera for subsequent analysis and refer to the orexin/Arch neurons as located within the LHA. Grouped data are expressed as mean \pm SEM.

Photoinhibition of Hcrt/orexin neurons in vivo. Arch can be activated by application of either yellow or green light to evoke neuronal silencing (Chow et al., 2010). To measure of the efficacy of Arch-mediated photoinhibition of Hcrt cells, green light illumination $(550 \pm 15 \mathrm{~nm})$ was delivered into the hypothalamus of WT $(n=11)$ and orexin/Arch $(n=$ 12) mice at ZT12 through the bilaterally implanted optical fibers (see In vivo optogenetics and tethered EEG recording) continuously for $1 \mathrm{~h}$. At ZT13, mice were killed for histology. Brain sections were processed for orexin-IR (guinea pig anti-orexin antiserum as described above) and rabbit anti-c-Fos antiserum (1:6000; AB5, Calbiochem). Affinipure donkey anti-guinea pig IgG (Vector Laboratories) and Affinipure donkey anti-rabbit IgG (Vector Laboratories) were used as secondary antibodies. Orexin-IR was detected with DAB (2 min; SK4100, Vector Laboratories); c-FOS was detected with $\mathrm{nDAB}$ (10 min; SK4100, Vector Laboratories).

Activation of Hcrt/orexin neurons in the chocolate-cataplexy assay (CCA). Mice implanted with EEG/EMG telemetry devices (see below) were permitted access to chocolate $(1 \times$ Hershey's kiss) from ZT12 to ZT16. At ZT16, mice were killed for histological examination (WT, $n=$ 8 ; aLE, $n=10$; aHE, $n=5$; ATAX, $n=5)$. Brain sections were processed for orexin-IR (combined anti-orexin-A and anti-orexin B antibodies as described above) and rabbit anti-c-FOS antiserum (1:2000; sc-52, Santa Cruz Biotechnology). Secondary antisera used were Affinipure donkey anti-goat IgG (1:1000, Jackson ImmunoResearch Laboratories) and Af- finipure donkey anti-rabbit IgG (1:1000, Jackson ImmunoResearch Laboratories). Orexin-IR was detected with DAB (2 min; SK4100, Vector Laboratories), and c-FOS was detected with nDAB (10 min; SK4100, Vector Laboratories).

\section{Ex vivo electrophysiology}

Recording of Hcrt/orexin neurons in vitro. Coronal brain slices $(250 \mu \mathrm{m})$ of the tuberal hypothalamus were prepared from juvenile (P14-P27) or adult ( $>8$ weeks) male and female orexin/Arch and orexin/eGFP (Yamanaka et al., 2003a,b) mice in ice-cold, oxygenated $\left(95 \% \mathrm{O}_{2}, 5 \% \mathrm{CO}_{2}\right)$ sucrose-based aCSF containing the following (in $\mathrm{mm}$ ): 250 sucrose, 2.5 $\mathrm{KCl}, 1.24 \mathrm{NaH}_{2} \mathrm{PO}_{4}, 10 \mathrm{MgCl}_{2}, 10$ glucose, $26 \mathrm{NaHCO}_{3}, 0.5 \mathrm{CaCl}_{2}(305$ $\mathrm{mOsm} / \mathrm{L}$ ). Slices were incubated in aCSF containing the following (in $\mathrm{mm}$ ): $124 \mathrm{NaCl}, 2.5 \mathrm{KCl}, 1.24 \mathrm{NaH}_{2} \mathrm{PO}_{4}, 1.3 \mathrm{MgCl}_{2}, 10$ glucose, 26 $\mathrm{NaHCO}_{3}, 2.5 \mathrm{CaCl}_{2}(300 \mathrm{mOsm} / \mathrm{L})$ at $37^{\circ} \mathrm{C}$ for $15 \mathrm{~min}$. Thereafter, slices were maintained and recorded at $22^{\circ} \mathrm{C}$ with aCSF flow rate of $\sim 1.5$ $\mathrm{ml} / \mathrm{min}$. For voltage- and current-clamp recordings, the pipette solution contained the following (in $\mathrm{mM}$ ): $130 \mathrm{~K}$-gluconate, $2 \mathrm{KCl}, 3 \mathrm{MgCl}_{2}, 2$ MgATP, 0.2 $\mathrm{Na}_{2}$ GTP, 10 HEPES, 0.2 EGTA (290 mOsm/L, pH 7.3). To measure sIPSC activity, the pipette solution contained the following (in mM): $145 \mathrm{CsCl}, 10$ HEPES, 1.1 EGTA, $0.3 \mathrm{CaCl}_{2}, 1 \mathrm{MgCl}_{2} \cdot \mathrm{H}_{2} \mathrm{O}, 0.5$ $\mathrm{Na}_{2}$ GTP $(290 \mathrm{mOsm} / \mathrm{L}$, pH 7.3 with $\mathrm{CsOH})$. All recordings were acquired with a MultiClamp 700A amplifier, Digidata 1322A digitizer interface and pClamp 9 software (Molecular Devices). Voltage-clamp data were sampled at $7 \mathrm{kHz}$ and filtered at $3 \mathrm{kHz}$; current-clamp data were sampled at $20-25 \mathrm{kHz}$ and filtered at $10 \mathrm{kHz}$. Changes in input resistance $\left(\mathrm{R}_{\text {in }}\right)$ were monitored across the experiments by injecting hyperpolarizing steps $(-20$ or $-40 \mathrm{pA})$ and periodically switching from voltageclamp mode to recording in current-clamp mode. Voltage-clamp recordings were then concatenated to remove breaks.

For current-clamp recordings, Hcrt/orexin neurons were recorded at their resting membrane potential (RMP). For voltage-clamp recordings, $V_{\mathrm{h}}$ was $-60 \mathrm{mV}$. Series resistance varied from 10 to $60 \mathrm{~m} \Omega$ and was monitored during voltage-clamp recordings. Any neurons deviating $>10 \%$ in series resistance over time were excluded from analysis; the bridge balance was maintained and monitored during current-clamp recordings. Membrane potential measurements were not corrected for the theoretical liquid junction potential of $-15 \mathrm{mV}$ (K-Glu intracellular) or $5 \mathrm{mV}$ ( $\mathrm{CsCl}$ intracellular) between pipette solution and bath solution. The reference electrode was a $\mathrm{Ag} / \mathrm{AgCl}^{-}$pellet.

Activation of Arch proton pump ex vivo. Whole-field illumination (40X objective) of yellow light ( $550 \pm 15 \mathrm{~nm}$ ) was generated by a SPECTRA III light engine (Lumencor).

\section{In vivo optogenetics and tethered EEG recording}

Optical fiber placement with EEG. Fifteen-week-old male orexin/Arch transgenic mice and their WT littermates were anesthetized with isoflurane using a vaporizer for small animals (400, Bio Research Center) and positioned in a stereotaxic frame (David Kopf Instruments). Plastic optical fibers ( $0.5 \mathrm{~mm}$ diameter; Eska, Mitsubishi Rayon) were bilaterally implanted $\sim 1 \mathrm{~mm}$ above the LHA $(1.2 \mathrm{~mm}$ posterior, $1 \mathrm{~mm}$ lateral from Bregma, $3.5 \mathrm{~mm}$ depth from brain surface). Electrodes for EEG and EMG were implanted on the skull and neck muscles, respectively, and were attached to the skull using dental cement (GC). The mice were then housed separately for a recovery period of at least $7 \mathrm{~d}$ before recording.

Continuous EEG and EMG recordings were performed through a slip ring (Air Precision) designed so that the movement of the mouse was unrestricted. EEG and EMG signals were amplified (AB-610J, Nihon Koden), filtered (EEG, 1.5-30 Hz; EMG, 15-300 Hz), digitized at a sampling rate of $128 \mathrm{~Hz}$, and recorded using SleepSign software version 3 (Kissei Comtec). Green light $(550 \pm 15 \mathrm{~nm})$ was generated by the SPECTRA III light engine (Lumencor) and applied through the plastic optical fibers. An optical swivel (COME2, Lucir) was used for unrestricted in vivo photoillumination. Green light power intensity at the tip of the plastic fiber optics ( $0.5 \mathrm{~mm}$ diameter) was measured with a power meter to be $0.46 \mathrm{~mW} / \mathrm{mm}^{2}$ (VEGA, Ophir Optronics). The animal's behavior was monitored through a CCD video camera and recorded on a computer synchronized with EEG and EMG recordings using the SleepSign video option (Kissei Comtec). 
In vivo validation of orexin/Arch transgenic mice. Three experiments were performed to assess the functionality of Arch in response to green light stimulation: (1) Green illumination was applied for a 1 min duration when mice were awake during both the inactive (ZT5-ZT11) and active phases (ZT13-ZT15). Each mouse received 1-3 illuminations, with a minimum intervening period of $4 \mathrm{~d}$ between photic stimulations. (2) If slow-wave activity was recorded within $30 \mathrm{~s}$ of initiation of photic stimulation, mice received tactile stimulation from an artist's brush to determine whether they could be aroused from the optogenetically induced sleep. (3) One hour continuous photoillumination was applied 6 times across the day/night cycle: at ZT12, ZT16, ZT20, ZT0, ZT4, and ZT8.

In vivo phenotyping of orexin/Arch transgenic mice

Implantation of EEG/EMG telemetry devices for basal sleep-wake analyses. Male orexin/Arch mice, orexin/Ataxin-3 (ATAX) mice (Hara et al., 2001), and WT littermates ( $>10$ weeks of age) were implanted with biotelemetry transmitters (F20-EET; Data Sciences International) for chronic recording of EEG, EMG, core body temperature $\left(\mathrm{T}_{\mathrm{b}}\right)$, and gross motor activity as previously described (Black et al., 2013, 2014, 2017b). Male mice implanted with telemeters were of the same age range (WT: $3.27 \pm$ 0.27 months, $n=9$; ATAX: $3.46 \pm 0.37$ months, $n=6$; aLE: $2.86 \pm 0.18$ months, $n=9$; aHE: $3.20 \pm 0.19$ months, $n=8$; one-way ANOVA, $\left.F_{(3,28)}=1.14, p=0.35\right)$ and similar body weight at the beginning of the study (WT: $32.11 \pm 1.60 \mathrm{~g}$; ATAX: $31.83 \pm 1.49 \mathrm{~g}, n=6$; aLE: $32.00 \pm$ 1.46 g; aHE: $37.00 \pm 1.85$ g; one-way ANOVA, $\left.F_{(3,28)}=2.35, p=0.09\right)$. Following a postsurgical recovery period, mice were singly housed with access to running wheels.

$S D$ procedures. For experiments related to sleep homeostasis, the reference time was lights-on (ZT0) when sleep pressure is high. To determine whether there were any differences in homeostatic sleep regulation across the 4 strains, mice were kept awake/sleep deprived for $4 \mathrm{~h}$ beginning at ZT0. SD was achieved by tapping the home cage and, when necessary, stroking the vibrissae or fur with an artist's brush to keep mice awake for the $4 \mathrm{~h}$ duration. To be included in the final analysis, mice must have been awake $>80 \%$ of the SD period.

Murine multiple sleep latency (mMSLT) test. To objectively measure sleepiness, we performed a variation of the MMSLT (Veasey et al., 2004) as previously described (Morairty et al., 2013). Starting $3 \mathrm{~h}$ into the light phase (ZT3), mice were subjected to five 20 min sessions of forced waking, each followed by a 20 min nap opportunity within a continuous 200 min time interval. The latencies to the onset of NREM and REM sleep as well as the percentage of time spent in each vigilance state were determined for each nap opportunity. To be included in the final analyses, mice must have been awake $>80 \%$ of each forced waking period.

Vigilance state and cataplexy assessments. As described below and in our previous publications (Black et al., 2013, 2014, 2017b; Schwartz et al., 2017), EEG and EMG data were classified in $10 \mathrm{~s}$ epochs as wakefulness, NREM, REM, or cataplexy. Criteria for cataplexy were $\geq 10 \mathrm{~s}$ of EMG atonia, theta-dominated EEG, and video-confirmed behavioral immobility preceded by $\geq 40$ s of wake (Scammell et al., 2009). All mice had access to a wheel. To facilitate the occurrence of cataplexy, we used the CCA in which mice were given $1 \times$ Hershey's kiss at ZT12 for a $24 \mathrm{~h}$ period (Oishi et al., 2013). Chow and chocolate consumption was measured during the assay to determine whether there was a taste preference between mouse strains. Two weeks before the CCA, all mice were given $1 \mathrm{~g}$ of a Hershey's kiss to acclimate mice to the smell and presentation of the chocolate. At the end of the basal sleep-wake phenotyping experiments, mice (WT, $n=8$; aLE, $n=10$; aHE, $n=5$; ATAX, $n=5$ ) were given $1 \times$ Hershey's kiss at ZT12 and killed for histology at ZT16.

Circadian assessment of wheel running activity. Mice were individually housed in polycarbonate cages equipped with running wheels. Mice were kept under the LD cycle for a minimum of $10 \mathrm{~d}$ and then released into constant darkness (DD) to establish stable free-running activity rhythms for at least 14 circadian cycles. Each revolution of the running wheel depressed an externally mounted microswitch, the activation of which was recorded via a PC using the collect module of the ClockLab (Actimetrics). Wheel revolution counts were analyzed and actograms were double-plotted using ClockLab software (Actimetrics). Endogenous circadian periods (tau) were calculated by $\chi^{2}$ periodogram in ClockLab.
Body weight and composition analysis. All mice were weighed during the course of the experiments to monitor body weight with respect to food intake (FI), wheel-running behavior, and general health. Cohorts of male and female mice of the four genotypes were killed to quantify fat deposition with respect to age. Subcutaneous fat and epididymal fat depots were dissected and weighed.

Analysis of FI and metabolism. Locomotion, FI, water intake, and respiratory exchange ratio (RER) were concurrently recorded using the Comprehensive Laboratory Animal Monitoring System (CLAMS; Columbus Instruments) as previously described (Inutsuka et al., 2014). Male mice ( 8 weeks old) were individually placed in CLAMS cages and monitored for $5 \mathrm{~d}$, the first $3 \mathrm{~d}$ of which were an acclimation period. Food and water consumption was measured directly, and $x / z$ ambulatory activity (a minimum of 3 different, consecutive horizontal beam breaks in counts) was used as the locomotion index. Data were collected every $10 \mathrm{~min}$.

Glucose tolerance test. At 9 weeks of age, male mice housed in the CLAMS were fasted overnight for $16 \mathrm{~h}$ and then injected with $2 \mathrm{~g} / \mathrm{kg}$ dextrose (i.p.) at ZT2. Blood glucose levels were measured using the ACCU-CHEK Nano meter (Roche Diagnostics). Blood samples were obtained by carefully pricking the tail vein using a sterile needle. Area under the curve was calculated with fasting glucose as the baseline.

\section{Experimental design and statistics}

In vitro electrophysiology. Patch-clamp recording data were analyzed using Clampfit 9 (Molecular Devices) and synaptic events using MiniAnalysis (Synaptosoft). The nonparametric Kolmogorov-Smirnov test (MiniAnalysis) was used to quantify the effects of photostimulation on sEPSC and sIPSC frequency for each group. Sample traces were generated with Igor Pro version 6 (WaveMetrics) and graphs produced with Prism 5 (GraphPad). For cell-attached recordings, spiking activity was averaged in $30 \mathrm{~s}$ bins. A baseline period of $4 \mathrm{~min}$ before photoillumination was averaged and compared with the same period length 1 min into light application, immediately upon light cessation, and 9 min after the end of the light application. $V_{\mathrm{m}}$ was determined in $30 \mathrm{~s}$ bins for a minimum period of $2 \mathrm{~min}$ before intervention. These data were then used to calculate the average baseline $V_{\mathrm{m}}$ or RMP. The effects of interventions on $V_{\mathrm{m}}$ were compared with this average baseline and the delta calculated.

For voltage-clamp analyses, cells were included if the peak current was greater than the mean \pm 2 SD of the preceding baseline value. To assess characteristics of glutamatergic events between genotypes, 200 events per baseline period were taken for analysis. Unless otherwise stated, data are presented as mean \pm SEM, with $n=$ number of cells per group (represented in parentheses for figures). We compared group means from the same cells using paired $t$ tests or one-way ANOVA followed by NewmanKeuls post hoc tests; changes over time were analyzed by repeatedmeasures ANOVA or two-way ANOVA followed by the Bonferroni or Newman-Keuls post hoc test; different groups of cells were then compared using unpaired $t$ tests.

Tethered EEG/EMG recordings. SleepSign software was used to score $4 \mathrm{~s}$ epochs of waking (W), NREM, and REM sleep according to standard criteria (Yamanaka et al., 2002). All vigilance state classifications assigned by SleepSign were confirmed following a visual examination by an independent scorer, blinded to genotype and experimental condition. Spectral analysis of the EEG was performed by fast Fourier transform (sampled at $128 \mathrm{~Hz}$ ), yielding a power spectra profile over a $0-40 \mathrm{~Hz}$ window with a $1 \mathrm{~Hz}$ resolution divided into delta $(1-5 \mathrm{~Hz})$, theta $(6-10$ $\mathrm{Hz})$, alpha $(10-13 \mathrm{~Hz})$, and beta $(13-25 \mathrm{~Hz})$ waves. An average EEG spectrum profile was calculated from EEG power densities in each frequency bin and then expressed as average power values in the delta, theta, alpha, and beta bands for each state. Data were analyzed by unpaired $t$ test, paired $t$ test, one-way ANOVA, or two-way ANOVA, as appropriate for the parameters examined, using KaleidaGraph 4.0 (Hulinks). When appropriate, ANOVA tests were followed by post hoc analysis of significance using Fisher's Protected Least Significant Difference test. $p<0.05$ was considered statistically significant.

Telemetry EEG/EMG recordings. EEG data from mice were scored in 10 s epochs by experts ( $\geq 96 \%$ interrater reliability) using NeuroScore 2.1 
A

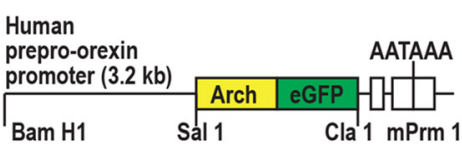

B
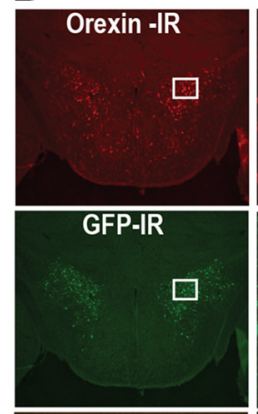

Merge 뭄
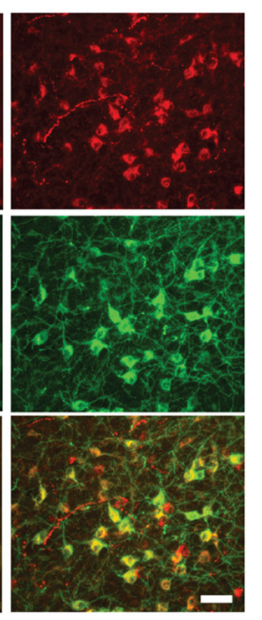

C
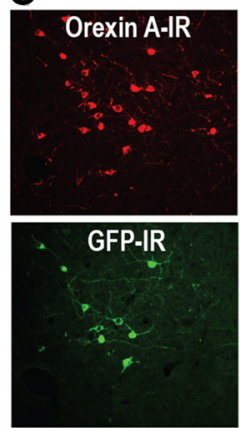

D

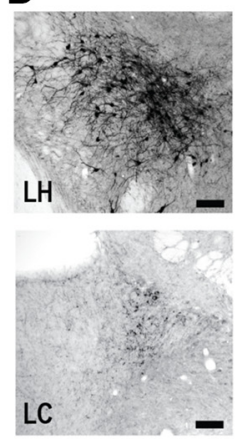

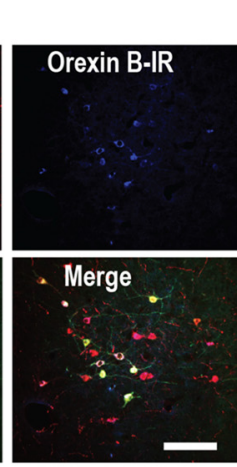

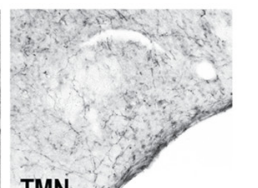

TMN

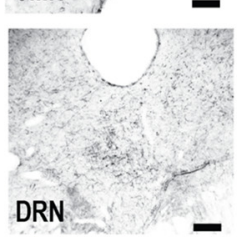

$\mathbf{E}$

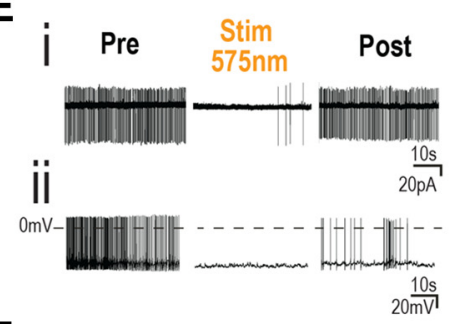

$\mathbf{F}$

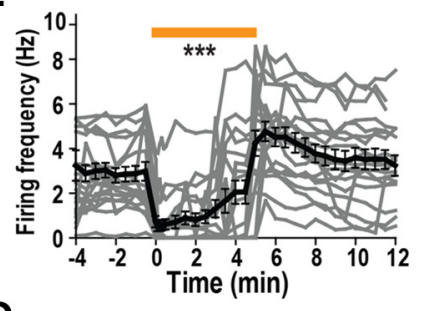

G
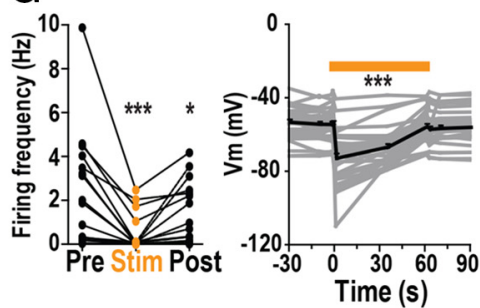

Figure 1. Archaerhodopsin (Arch) expression in Hcrt/orexin neurons in the orexin/Arch transgenic mouse brain. $\boldsymbol{A}$, Schematic of transgene used to express Arch downstream of the prepro-orexin promoter. B, Top, Immunostaining of Hcrt/orexin-IR neurons (Alexa-594, red) located in the LHA. Middle, GFP-IR neurons (Alexa-488, green), reporting Arch expression, are also observed in the LHA. Bottom, Merged picture showing expression of Arch within Hcrt/orexin neurons in the LHA of the orexin/Arch transgenic mice. Scale bars: left column, $300 \mu \mathrm{m}$; right column, $50 \mu \mathrm{m}$. Arch expression was not observed in nonorexin neurons in any brain region. $C$, Arch expression was observed in the dendrites as well as somata of identified orexin-A-IR (red channel) and orexin-B-IR neurons (blue channel). Scale bar, $50 \mu \mathrm{m}$. Approximately $80 \%$ of Hcrt/orexin neurons colocalized with Arch. $\boldsymbol{D}$, Immunohistochemistry demonstrates Arch expression in orexin-innervated regions, such as the tuberomammillary nucleus (TMN), locus coeruelus (LC), and dorsal raphe nucleus (DRN). $E$, In vitro functionality and sensitivity of the Arch proton pump expressed in $\mathrm{Hcrt} /$ orexin cells of orexin/Arch mice. Representative examples demonstrating that $575 \pm 25 \mathrm{~nm}$ illumination of $\mathrm{Hcrt} /$ orexin neurons from orexin/Arch mice results in a reduction in firing rate in both (Ei) cell-attached and (Eii) current-clamp configurations. F, Yellow light stimulation ( $575 \pm 25 \mathrm{~nm}$, orange bar) significantly reduced the firing activity of orexin/Arch neurons in cell-attached configuration. $\mathbf{G}$, Photoactivation of Arch similarly reduced electrical firing with concomitant hyperpolarization of the membrane potential in current-clamp recordings of Hcrt/orexin neurons. ${ }^{*} p<0.05$; ${ }^{* * *} p<0.001$. Values are mean \pm SEM

(Data Sciences International). Epochs were classified as wakefulness (mixed-frequency, low-amplitude EEG and high-amplitude, variable EMG); wakefulness with wheel-running; REM sleep (theta-dominated EEG and EMG atonia); NREM sleep (low-frequency, high-amplitude EEG and low-amplitude, steady EMG); or cataplexy. Data were analyzed as time spent in each state. To assess sleep intensity, EEG spectra during NREM sleep were computed using the fast Fourier transform algorithm in NeuroScore (Data Sciences) on all $10 \mathrm{~s}$ epochs without visually detectable artifact. EEG delta power $(0.5-4 \mathrm{~Hz})$ in NREM sleep (NRD) was calculated. NRD during the recovery after SD was normalized to each animal's average NRD during the $24 \mathrm{~h}$ baseline recording. Wheel-running activity (OA) was classified if mice spent $>90 \%$ of an epoch running in the wheel. OA was included in percent time calculations for wakefulness, but OA epochs were excluded from the spectral analysis of wake due to high theta activity. NREM sleep latency was determined as the time between light onset and the first 1 , 3 , or 6 consecutive epochs of NREM sleep; REM sleep latency was determined as the time between light onset and the first 1, 2, or 3 consecutive epochs of REM sleep. When ANOVA indicated significance, contrasts between relevant factor levels were detected with post hoc Bonferroni $t$ tests with $\alpha=0.006$ (see Table 4) and $\alpha=0.02$ (see Fig. $5 C$ ) to correct for multiple comparisons.

\section{Results}

Specific expression of Arch in Hcrt/orexin neurons

Transgenic mice were generated in which Hcrt/orexin neurons expressed Arch under control of the human prepro-orexin promoter (orexin/Arch transgenic mice). This $3.2 \mathrm{~kb}$ DNA fragment
(Fig. 1A) has been used previously to express exogenous genes in the Hcrt/orexin neurons (Sakurai et al., 1999, 2005; Hara et al., 2001; Yamanaka et al., 2003a; Tsujino et al., 2005). Since Arch was expressed as a fusion protein with GFP, Arch expression in Hcrt/orexin neurons was confirmed by double-labeled immunohistochemistry using an anti-GFP antibody. Only 1 of 12 transgenic orexin/Arch mouse lines (line D3) expressed Arch in Hcrt/ orexin neurons; consequently, line D3 was used for all subsequent in vitro and in vivo experiments. A merged picture of GFP-IR and orexin-IR revealed that Arch was exclusively observed in $78.5 \pm 1.9 \%$ of the total orexin-IR population in line D3 $(n=3$; Fig. $1 B)$. In this population, Arch was found in $81.9 \pm$ $2.2 \%$ of orexin-A-IR cells ( $n=5$; Fig. $1 C$, red channel) and $85.3 \pm$ $2.9 \%$ of the orexin-B-IR-expressing cells $(n=5$; Fig. $1 C$, blue channel). Arch expression (Fig. 1B, $C$, green channel) was not found in any other cell type within the brain. GFP-IR was also present in the dendrites as well as somata of Hcrt/orexin neurons. Hcrt/orexin neurons expressing Arch did not show blebbing or other features indicative of inappropriate trafficking.

We did not find any differences in the total number of orexin-IR neurons between mice from the orexin/Arch D3 line and WT mice (data not shown). Analysis of key downstream targets of the Hcrt/ orexin neurons (the locus coeruleus (LC), tuberomammillary nucleus, and dorsal raphe nucleus) demonstrated Arch colocalization in the terminal fields of orexin-expressing neurons (Fig. 1D). 


$\begin{aligned} & \text { Table 1. Average episode duration and total time of each vigilance state recorded } \\
& \text { from transgenic orexin/Arch and littermate WT mice }{ }^{a}\end{aligned}$
\begin{tabular}{llcc}
\hline Parameter & State & WT mice $(n=5)$ & Orexin/Arch mice $(n=5)$ \\
\hline Light phase & & & \\
Episode duration (s) & Wake & $146.6 \pm 10.4$ & $128.5 \pm 15.3$ \\
& NREM & $279.2 \pm 27.1$ & $296.0 \pm 33.3$ \\
& REM & $96.7 \pm 7.6$ & $83.2 \pm 4.6$ \\
Total time (min) & Wake & $231.4 \pm 18.7$ & $200.2 \pm 8.5$ \\
& NREM & $434.1 \pm 16.9$ & $464.9 \pm 8.9$ \\
& REM & $54.5 \pm 2.3$ & $54.9 \pm 6.3$ \\
Dark phase & & & \\
Episode duration (s) & Wake & $426.9 \pm 81.6$ & $322.2 \pm 75.8$ \\
& NREM & $362.9 \pm 87.8$ & $259.3 \pm 36.3$ \\
& REM & $84.4 \pm 4.2$ & $77.2 \pm 4.5$ \\
Total time (min) & Wake & $399.1 \pm 36.2$ & $372.1 \pm 25.7$ \\
& NREM & $288.2 \pm 31.5$ & $316.8 \pm 20.9$ \\
& REM & $32.8 \pm 5.9$ & $31.1 \pm 5.2$ \\
\hline
\end{tabular}

$\overline{{ }^{a} \text { Values are mean } \pm \text { SEM. Wake, Wakefulness; NREM, non-Rapid Eye Movement Sleep; REM, Rapid Eye Movement }}$ Sleep.

\section{In vitro functionality of Arch-expressing Hcrt/orexin neurons}

To confirm that photoactivation of Arch silenced Hcrt neurons, whole-cell patch-clamp recordings from ex vivo brain slices were performed. Both male and female juvenile (P14-P27) and adult ( $>8$ weeks of age) mice were studied. Application of whole-field yellow light $(575 \pm 25 \mathrm{~nm}$ ) decreased firing activity in both cellattached and current-clamp recording configurations (Fig. 1E). Quantification of cell-attached spiking activity from orexin/Arch neurons indicated a basal firing rate of $2.95 \pm 0.05 \mathrm{~Hz}(n=20$; Fig. $1 F)$, which was significantly reduced during photoillumination to $1.29 \pm 0.19 \mathrm{~Hz}$ (one-way ANOVA, $F_{(3,19)}=6.79, p<$ $0.0001)$. A rebound in spiking activity occurred after the termination of light $(4.23 \pm 0.12 \mathrm{~Hz}, p=0.01)$ before resuming a steady-state firing rate not dissimilar to baseline $(3.58 \pm 0.4 \mathrm{~Hz})$. In current clamp (Fig. 1G), Hcrt/orexin neurons exhibited an inhibitory response in both firing frequency and membrane potential $\left(V_{\mathrm{m}}\right)$ in response to photoillumination. Prelight application firing frequency averaged $2.46 \pm 0.6 \mathrm{~Hz}(n=17)$, which was significantly reduced to $0.45 \pm 0.2 \mathrm{~Hz}$ (one-way ANOVA, $\left.F_{(2,16)}=4.59, p=0.0001\right)$. Post phototillumination firing frequency was $1.32 \pm 0.34 \mathrm{~Hz}$. $V_{\mathrm{m}}$ was $-54.07 \pm 1.43 \mathrm{mV}$ before yellow light application and significantly hyperpolarized to $-72.87 \pm 2.48 \mathrm{mV}(n=33)$ at yellow light initiation, reaching a steady state of $-66.88 \pm 1.72 \mathrm{mV}$ (one-way ANOVA, $F_{(4,32)}=$ 9.29, $p<0.0001$; Fig. $1 F$ ). Upon cessation of yellow light, $V_{\mathrm{m}}$ returned to prestimulation levels $(-55.73 \pm 1.48 \mathrm{mV})$. These data demonstrate efficient optical inhibition of Hcrt/orexin neuronal excitability in orexin/Arch mice.

\section{In vivo photoinhibition of Hcrt/orexin neurons induces NREM sleep}

Having demonstrated that Arch activation in vitro inhibits the electrical activity of Hcrt/orexin neurons, we next examined the effects of in vivo bilateral photoinhibition of these neurons on sleep-wake states. First, the spontaneous sleep-wakefulness pattern of orexin/Arch transgenic mice was analyzed to determine whether the presence of the Arch transgene affected normal sleep-wakefulness regulation. We compared the sleep-wakefulness episode durations and total time in each state in freely moving orexin/Arch transgenic mice to WT littermate mice. Neither the episode duration nor total time in state differed significantly between the two genotypes in either the light or dark phases (Table 1). These results suggest that sleep-wake- fulness patterns were unaffected by Arch expression in Hcrt/ orexin neurons.

To study the physiological significance of Hcrt/orexin neuronal activity in the regulation of sleep-wakefulness, we performed in vivo photic illumination of the LHA in freely moving orexin/ Arch transgenic mice. When green light $(555 \pm 28 \mathrm{~nm})$ from an LED light source was delivered to the LHA of orexin/Arch mice for $60 \mathrm{~s}$ during periods of spontaneous wakefulness in the second half of the light phase (13:00-19:00), EEG power gradually increased and EMG power gradually decreased, indicative of the onset of NREM sleep (Fig. 2A). During the second half of the $60 \mathrm{~s}$ photoillumination period, all orexin/Arch transgenic mice showed NREM sleep ( $n=13$; Fig. 2 Aiii); REM sleep was not observed during this brief illumination period (Fig. 2Aiv). Within a few seconds after termination of photic illumination, the EEG showed fast waves and increased EMG levels occurred, indicative of wakefulness (Fig. 2Ai). After a brief period of wakefulness (Fig. 2Aii), mice tended to fall asleep again without any further activation of Arch via photoillumination (Fig. 2Aiii). In contrast, when the same photoillumination paradigm was applied to bilaterally implanted WT littermates, no observable changes in sleep-wake parameters were recorded (data not shown). Furthermore, green light illumination of the LHA of freely moving orexin/Arch transgenic mice $(n=9)$ had no effect on sleep or wakefulness during the dark phase (21:00-23:00; ZT13-ZT15) when nocturnal mice have high levels of spontaneous activity and arousal.

Since mice can normally be awakened by tactile stimulation during spontaneous sleep, we evaluated whether orexin/Arch mice were similarly sensitive during photoillumination-induced NREM sleep. Approximately $50 \mathrm{~s}$ after initiating photoillumination in the light phase between ZT7-ZT9, orexin/Arch transgenic mice were stroked with an artist's brush during green lightinduced NREM sleep. As soon as orexin/Arch mice were stimulated, desynchronized activity occurred in the EEG and increased EMG levels were observed, indicative of an awakening from sleep. All orexin/Arch transgenic mice $(n=9)$ showed transitions from NREM to wakefulness after tactile stimulation. These results indicate that the cessation of activity induced by photic illumination is different from paralysis and similar to physiological NREM sleep.

\section{Efficacy of in vivo green light illumination to change sleep-} wake states in orexin/Arch mice is dependent upon time of day Having determined that acute (1 min) photoinhibition of Hcrt/ orexin neurons in orexin/Arch mice can induce NREM sleep, we evaluated whether prolonged $(1 \mathrm{~h})$ illumination would also be effective and whether any effects observed varied across the $24 \mathrm{~h}$ period. In contrast to brief $1 \mathrm{~min}$ photoillumination, green illumination of the LHA for $1 \mathrm{~h}$ periods delivered during the light phase from ZT0-ZT1, ZT5-ZT6, or ZT8-ZT9 (Fig. 2Bi) did not significantly affect arousal states relative to control (Fig. 2Bii). In contrast, when green illumination was delivered to the LHA just after dark onset (ZT12-ZT13) or 4 h later from ZT16-ZT17 (Fig. $2 \mathrm{Ci}$ ), the time spent awake decreased (Fig. 2Cii; basal $39.4 \pm 2.4$ $\min$ vs stim $25.6 \pm 2.7 \mathrm{~min}, n=10 ; p=0.0004$, paired $t$ test) and the time in NREM increased (basal $19.7 \pm 2.2 \mathrm{~min}$, stim $32.8 \pm$ $2.4 \min , n=10 ; p<0.0001$, paired $t$ test). The EEG spectra during photoillumination of orexin/Arch mice was indistinguishable from sleep-wake of WT mice (Fig. 2-1, available at https:// doi.org/10.1523/JNEUROSCI.0311-19.2019.f2-1). Locomotor activity (LMA) also decreased during light stimulation and the number of the transitions between states increased (Fig. 2Cii). 

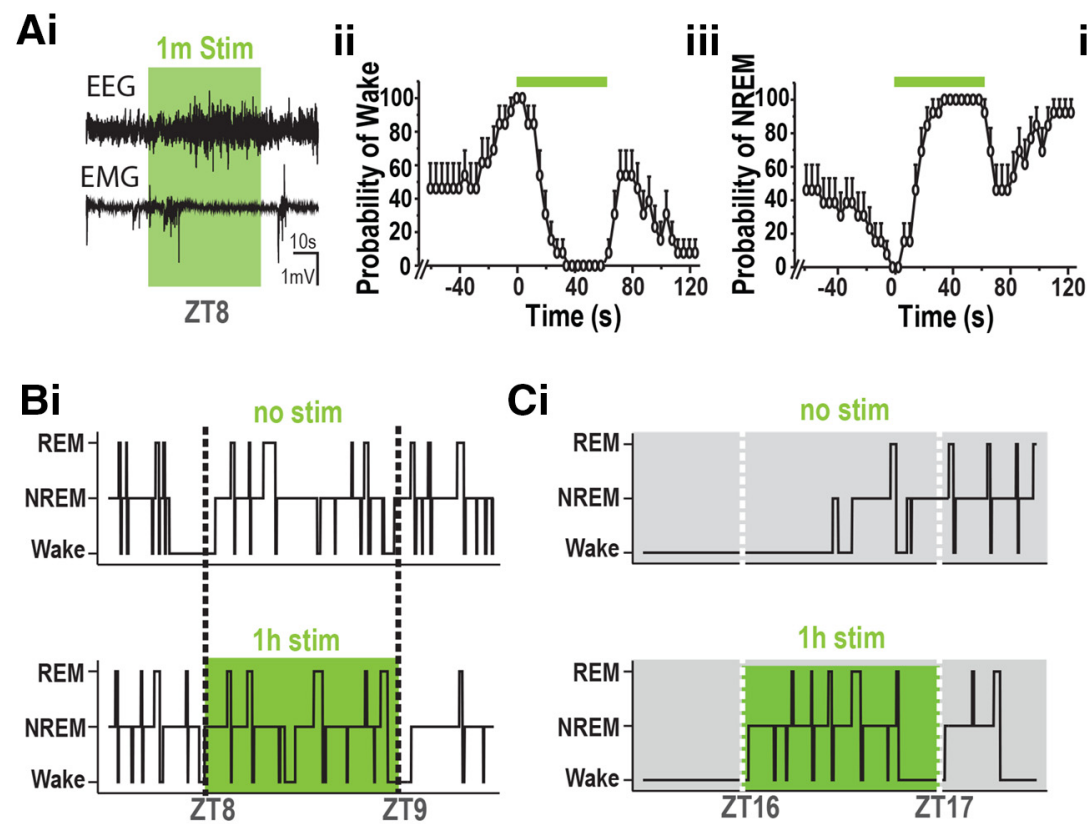

Ci
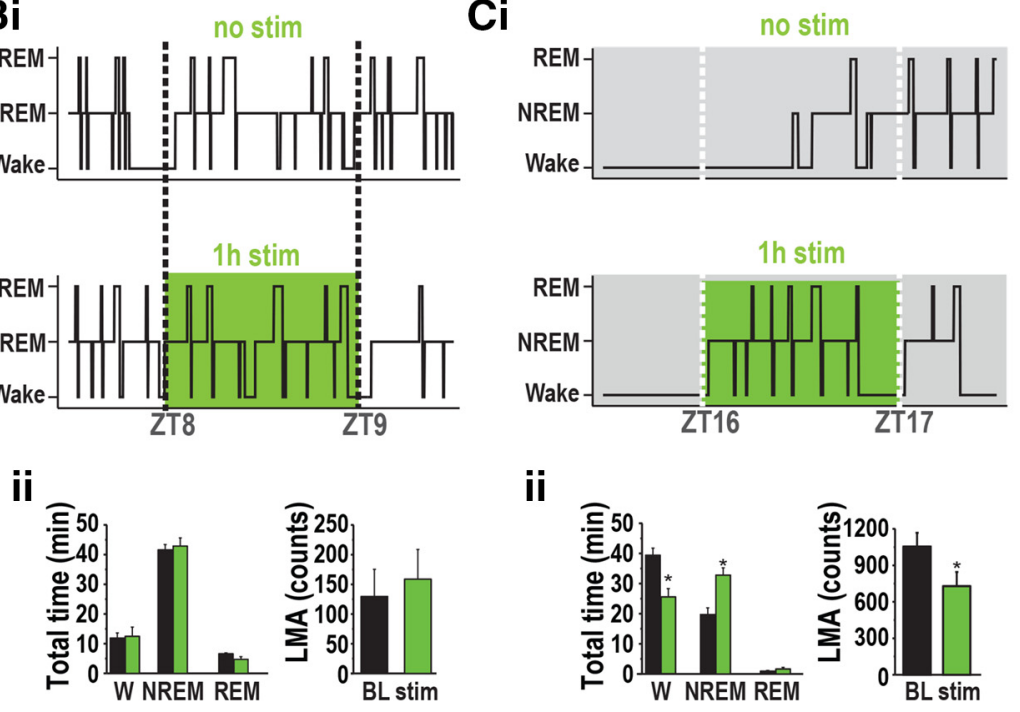

ii

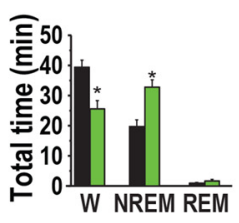

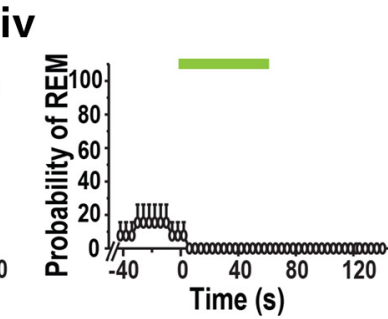
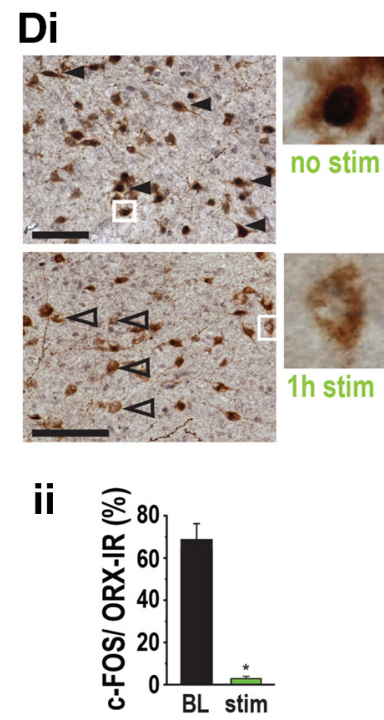

Figure 2. Bilateral in vivo green light stimulation activates Arch and silences Hcrt/orexin neurons. Ai, Acute 1 min photoillumination applied at random intervals during wake bouts between ZT8 and ZT11 decreased the probability of wake (Aii) and increased NREM sleep (Aiii) but not REM sleep (Aiv). Bi, Longer $1 \mathrm{~h}$ photoillumination between ZT8 and ZT9 did not change the total time spent in sleep versus wake (Bii) compared with baseline conditions. $\mathbf{C}$, In contrast, when $1 \mathrm{~h}$ green light was applied between ZT16 and ZT17, a significant increase in NREM sleep and concomitant reductions in both wake and LMA (Cii) occurred during photoillumination. The EEG spectra during photoillumination at any time of day were indistinguishable from sleep-wake of WT mice (Fig. 2-1, available at https://doi.org/10.1523/JNEUROSCI.0311-19.2019.f2-1). Di, One hour photoillumination beginning at ZT12 resulted in a decrease of c-FOS in orexin-IR neurons. Top, Black triangles represent double-labeled c-FOS ${ }^{+}$Hcrt/orexin neurons. Bottom, Open triangles represent c-FOS ${ }^{-}$Hcrt/orexin neurons. Open triangles represent orexin ${ }^{-}$neurons. Dii, Bar graph represents the percentage of Fos ${ }^{+}$orexin neurons ( $n=11$ for control, $n=12$ for light illumination). Values are mean \pm SEM. ${ }^{*} p<0.05$ versus prelight application.

Green light stimulation delivered toward the end of the dark phase from ZT20-ZT21 was ineffective, as during the light phase.

\section{Inhibition of Hcrt/orexin neuronal activity by green light illumination in vivo}

To determine whether green light activation of Arch affected Hcrt/orexin neurons in vivo, we examined c-FOS expression in Hcrt/orexin neurons. We compared orexin/Arch transgenic mice exposed with $1 \mathrm{~h}$ green light stimulation beginning at ZT12 $(n=$ $6)$ and sham (nonilluminated) controls $(n=6)$ (Fig. 2Di). The proportion of orexin-IR neurons expressing c-FOS-IR was significantly reduced in orexin/Arch mice subjected to green light illumination (Fig. 2Dii; controls $69.6 \pm 6.1 \%$, illuminated $2.6 \pm$ $1.1 \% ; p<0.0001$, unpaired $t$ test), demonstrating a robust reduction in Hcrt neural activity during illumination.

\section{Arch expression levels change the sensitivity to photoillumination}

qPCR reflecting transgene copy number distinguished two subgroups of orexin/Arch mice: low (aLE) or high (aHE) Arch expressers (Fig. 3A). Analysis was performed to determine whether age (juvenile: P14-P27; adult: $>8$ weeks) and transgene expression level contributed to the efficacy of photoinhibition on Hcrt/ orexin neuron activity and behavior. We hypothesized that higher Arch expression levels, reflected by higher qPCR values, would be more effective in silencing Hcrt/orexin neurons when photoilluminated. Cell-attached electrophysiological recordings did not distinguish significant differences in prestimulation spiking activity among the groups (adult aLE: $2.14 \pm 0.07 \mathrm{~Hz}, n=3$; juvenile aLE: $3.07 \pm 0.58 \mathrm{~Hz}, n=7$; adult aHE: $3.71 \pm 0.70 \mathrm{~Hz}$, $n=5$; juvenile aHE: $2.34 \pm 0.67 \mathrm{~Hz}, n=6$; one-way ANOVA, $\left.F_{(3,17)}=1.05, p=0.39\right)$. Upon 5 min photoillumination, however, Hcrt/orexin neuron firing frequency was significantly reduced in all groups, except juvenile aLE mice in which Hcrt/ orexin neuronal firing began to disinhibit within $\sim 3-4 \mathrm{~min}$ despite continued light stimulation (Fig. 3B). Upon cessation of photoillumination, spiking activity rebounded in all groups (adult aLE: $3.22 \pm 0.05 \mathrm{~Hz}, n=3$; juvenile aLE: $4.33 \pm 0.09 \mathrm{~Hz}$, $n=7$; adult aHE: $5.16 \pm 0.22 \mathrm{~Hz}, n=5$; juvenile aHE: $3.75 \pm$ 0.11-hz, $n=6$; one-way ANOVA, $\left.F_{(15,7)}=62.08, p<0.0001\right)$ before returning to prestimulation levels.

In current clamp, photoillumination significantly hyperpolarized the $V_{\mathrm{m}}$ of Hcrt/orexin neurons in all orexin/Arch mice tested (Fig. $3 C$; adult aLE: $\Delta=-39.48 \pm 2.52 \mathrm{mV}, n=5$; juvenile aLE: $\Delta=-18.85 \pm 4.38 \mathrm{mV}, n=10$; adult aHE: $\Delta=-13.76 \pm$ $1.18 \mathrm{mV}, n=11$; juvenile aHE: $\Delta=-16.08 \pm 2.08 \mathrm{mV}, n=7$; two-way ANOVA, $p<0.001)$. In addition, yellow light illumination reduced the firing frequency in aLE mice (Fig. 3D; adult: baseline $3.37 \pm 0.4 \mathrm{~Hz}$ vs light pulse $0.25 \pm 0.2 \mathrm{~Hz}$, two-way repeated-measures ANOVA, $p<0.001$; juvenile: baseline $3.88 \pm$ $1.33 \mathrm{~Hz}$ vs light pulse $1.19 \pm 0.5 \mathrm{~Hz}$; two-way repeated-measures ANOVA, $p<0.001)$. Unexpectedly, aHE mice had lower baseline 
A

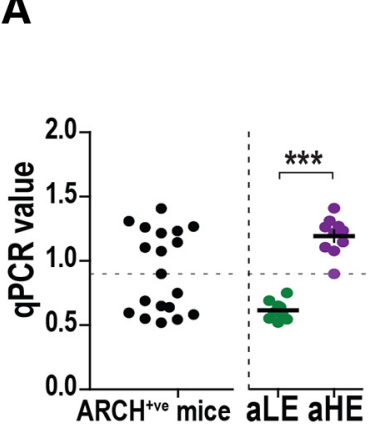

B Pre vs
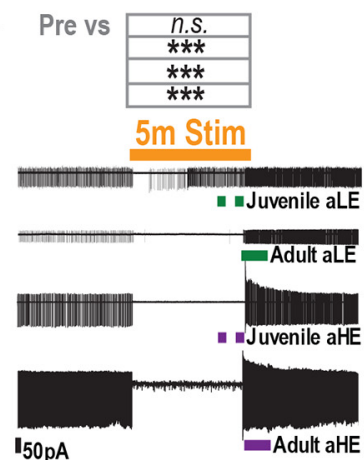

C

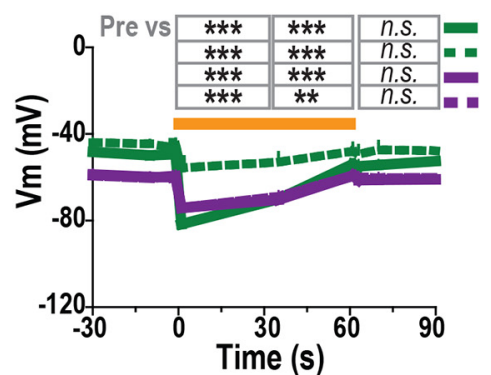

D

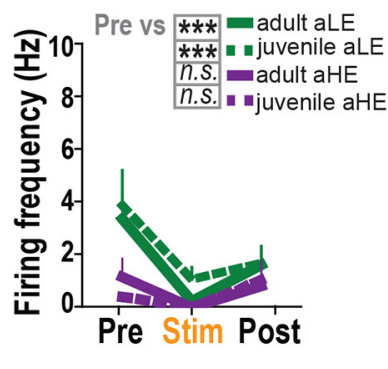

E

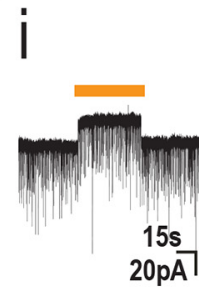

F

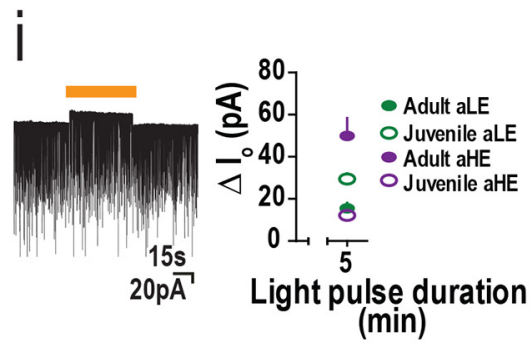

G

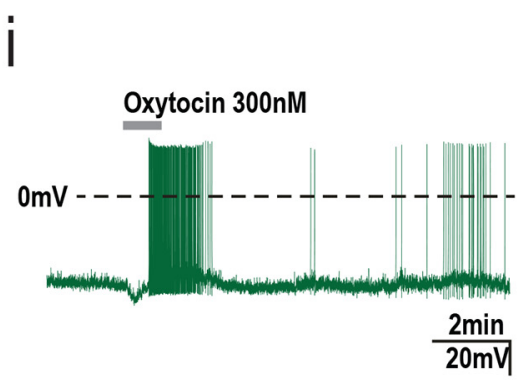

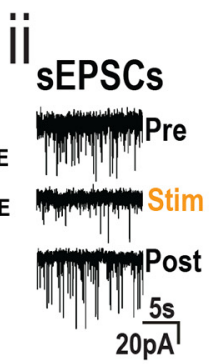

ii

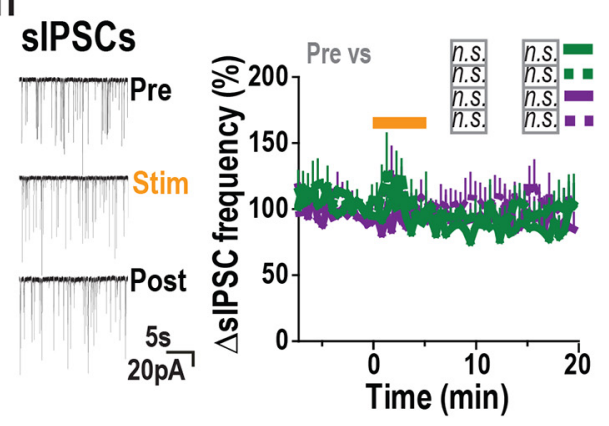

iii
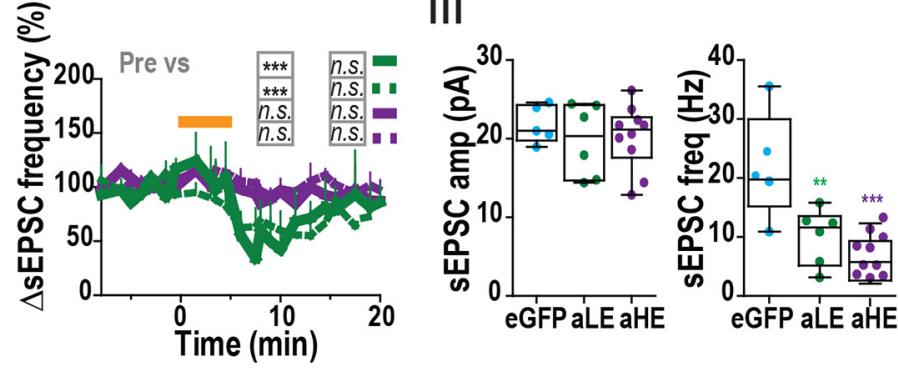

iii

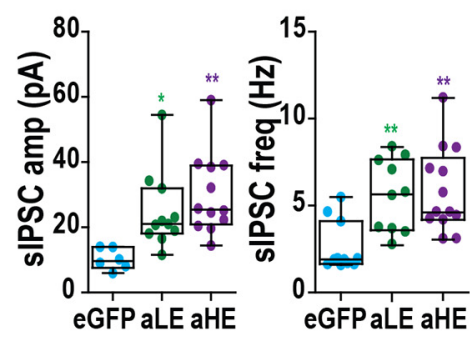

ii

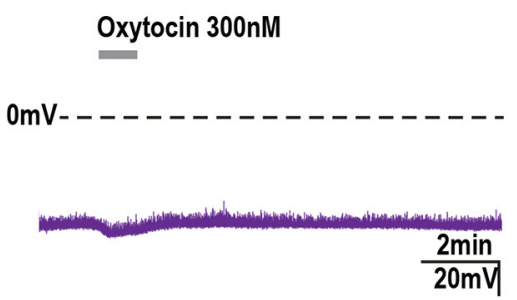

Figure 3. Differential Arch expression levels in orexin/Arch mice cause aberrant electrophysiological properties of Hcrt/orexin neurons and result in a gradient of photoinhibition. $A, q P C R$ results distinguished two subgroups of orexin/Arch mice, low- (aLE) or high- (aHE) Arch expressers, based on gene copy number. $B$, Cell-attached recordings in response to 5 min photoillumination (orange line, $575 \pm 25 \mathrm{~nm}$ ) grouped by age (juvenile: P14-P27; adult: $>8$ weeks) and Arch expression. In contrast to juvenile aLE mice, adult orexin/Arch mice exhibited photoinhibition throughout the illumination period. Upon cessation of photoillumination, there was a significant rebound in spiking activity, before the firing rate returned toward prestimulation levels. C, Summary data indicate that photoillumination silenced Hcrt/orexin neuronal activity. However, Hcrt/orexin neurons in aHE mice of both ages (purple) had hyperpolarized $\operatorname{RMPs}\left(V_{m}\right)$ and $(\boldsymbol{D})$ a lower basal firing rate. $\boldsymbol{E}$, Photoillumination evoked a significant outward current $\left(\mathrm{I}_{0}\right)$ with different light durations ( $\boldsymbol{E}$ i). Photoillumination also decreased sEPSCS onto Hcrt/orexin neurons in aLE mice (Eii). Further characterization of basal sEPSC activity (Eiii) indicated no difference in sEPSC amplitude between Hcrt/orexin neurons from orexin/Arch and orexin/eGFP mice, although there was a significant reduction in the frequency of glutamatergic events that was more pronounced in aHE mice. $\boldsymbol{F}$, When photoillumination was applied in conditions to measure sIPSCS, yellow light $(575 \pm 25 \mathrm{~nm}$ ) evoked an outward current in all groups (Fi), but sIPSC activity did not change (Fii). Nevertheless, basal GABAergic tone was altered in orexin/Arch mice relative to orexin/eGFP mice, with a larger amplitude of basal events and greater sIPSC frequency (Fiii). $\mathbf{G}$, Bath application of oxytocin (300 nm), a known activator of Hcrt/orexin neurons, had differential effects on the excitability of Hcrt/orexin neurons in (Gi) aLE and (Gii) aHE orexin/Arch mice: oxytocin significantly depolarized $V_{\mathrm{m}}$ of $\mathrm{Hcrt} /$ orexin neurons from aLE mice but not aHE mice (Giii). Values are mean \pm SEM. ${ }^{*} p<0.05,{ }^{* *} p<0.01,{ }^{* * *} p<0.001$; n.s., non-significant as in legends to Figures 4 and 5. 
firing rates than aLE mice (adult: $1.16 \pm 0.7 \mathrm{~Hz}, n=4$; juvenile: $0.40 \pm 0.2 \mathrm{~Hz}, n=4$; one-way ANOVA, $p=0.03$ ) and yellow light completely inhibited firing, yet statistical significance was not reached (two-way ANOVA, $p>0.05$ ). Overall, the RMP of aLE mice was comparable with Hcrt/orexin neurons of orexin/ eGFP mice (aLE: $-46.59 \pm 1.20 \mathrm{mV}, n=15$; eGFP: $-51.30 \pm$ $2.68 \mathrm{mV}, n=5$; one-way ANOVA, $p>0.05$ ), but neurons from aHE mice were significantly hyperpolarized (aHE: $-59.89 \pm 1.38$ $\mathrm{mV}, n=22 ; p<0.01$ ), even in the absence of yellow light illumination. Nonorexin neurons, which did not have GFP fluorescence, showed no response to yellow light illumination in either orexin/EGFP or orexin/Arch mice (data not shown).

\section{Yellow light illumination induces an outward current in Arch-expressing Hcrt/orexin neurons}

Since yellow light illumination reduced the $V_{\mathrm{m}}$ and inhibited firing of Arch-expressing Hcrt/orexin neurons, we assessed the magnitude of light-evoked current. In voltage clamp, yellow light stimulation of different durations (1,2, and $5 \mathrm{~min}$ ) evoked an outward current in all groups tested, although age and transgene expression levels affected the magnitude of the current (Fig. 3Ei). Juvenile aLE mice had the smallest average current evoked $(10.99 \pm 1.60 \mathrm{pA}, n=21)$. In contrast, photo-induced currents in juvenile aHE mice $(26.44 \pm 1.84 \mathrm{pA}, n=68)$ were significantly greater (one-way ANOVA $F_{(3,156)}=5.07, p=0.02$ ) and comparable with those of Hcrt/orexin neurons from adult aLE mice $(24.33 \pm 2.68 \mathrm{pA}, n=19)$ yet significantly lower than for adult aHE recordings $(52.86 \pm 2.90 \mathrm{pA}, n=52$; Fig. $3 \mathrm{Ei})$. These results suggest that the magnitude of the photoinduced currents evoked is related to the degree of Arch expression in Hcrt/orexin neurons.

\section{Yellow light illumination differentially affects glutamatergic inputs to Hcrt/orexin neurons in aLE versus aHE mice}

Since Hcrt neurons are thought to release glutamate and mutually innervate each other, we next determined the effect of yellow light stimulation on glutamatergic tone (Fig. 3Eii). The frequency of sEPSCs was significantly reduced following yellow light stimulation in aLE Hcrt/orexin neurons (juvenile: $\Delta=-35.21 \pm$ $0.82 \%, n=3$; adult: $\Delta=-55.72 \pm 4.8 \%, n=3$; one-way ANOVA, $p<0.01$ and $p<0.001$, respectively). In contrast, this response was not seen in either aHE age group.

\section{Photoinhibition of Hcrt/orexin neurons does not affect GABAergic tone}

We also analyzed the effect of photoillumination on Archexpressing neurons in relation to sIPSCs (Fig. 3F). Under these conditions, yellow light inhibition evoked significant outward currents in all groups (juvenile aLE: $\Delta 12.20 \pm 2.41 \mathrm{pA}, n=5$; adult aLE: $\Delta 15.52 \pm 2.63 \mathrm{pA}, n=5$; juvenile aHE: $\Delta 29.45 \pm 2.73$ pA, $n=8$; adult aHE: $\Delta 49.92 \pm 8.44 \mathrm{pA}, n=6$; one-way ANOVA, $F_{(3,20)}=11.68, p=0.0001$; Fig. 3Fi), but the change in sIPSC frequency did not differ across groups (Fig. 3Fii). Thus, unlike sEPSC activity, photoillumination of Arch in Hcrt/orexin neurons did not affect sIPSC activity.

\section{Increased expression of Arch affects basal properties of Hcrt/ orexin neurons}

As discussed above, the Hcrt/orexin neurons of aHE mice had significantly hyperpolarized RMP and decreased basal firing rates yet exhibited the largest change in $V_{\mathrm{m}}$ and outward current during photoillumination and activation of Arch. Therefore, we determined whether Arch transgene expression modified Hcrt/ orexin neuronal function without direct activation of Arch by photoillumination. Further analysis of sEPSC basal tone (Fig. 3 Eiii) indicated that sEPSC event amplitude was similar in orexin/ eGFP $(21.83 \pm 1.07 \mathrm{pA}, n=5)$ and orexin/Arch mice (aLE: $19.76 \pm 1.89 \mathrm{pA}, n=6$; aHE: $20.23 \pm 1.28 \mathrm{pA}, n=10$; one-way ANOVA, $\left.F_{(2,18)}=1.63, p=0.44\right)$. However, Arch expression significantly reduced sEPSC frequency, particularly in Hcrt/ orexin neurons from the aHE cohort (eGFP: $22.02 \pm 4.02 \mathrm{~Hz}$, $n=5$; aLE: $10.14 \pm 1.93 \mathrm{~Hz}, n=6$; aHE: $6.21 \pm 1.13, n=10$; one-way ANOVA, $\left.F_{(2,18)}=13.72, p=0.0002\right)$. These observations may explain why photoinhibition of sEPSC activity was only found in Hcrt neurons from aLE mice (i.e., there was a floor effect in cells from aHE mice).

In addition, sIPSC amplitude (Fig. 3Fiii) was significantly increased in orexin/Arch mice (eGFP: $10.23 \pm 1.32 \mathrm{pA}, n=6$; aLE: $24.81 \pm 3.55 \mathrm{pA}, n=11$; aHE: $30.05 \pm 3.55 \mathrm{pA}, n=12$; one-way ANOVA, $\left.F_{(2,26)}=7.61, p=0.02\right)$. A significant increase in sIPSC frequency also occurred (eGFP: $2.60 \pm 0.43 \mathrm{~Hz}, n=11$; aLE: $5.56 \pm 0.66 \mathrm{~Hz}, n=10$; aHE: $5.83 \pm 0.67 \mathrm{~Hz}, n=13$; one-way ANOVA, $\left.F_{(2,31)}=8.63, p=0.001\right)$, suggesting a change in the excitatory properties of Hcrt/orexin neurons. Together, these changes in basal excitatory-inhibitory drive in the absence of photoillumination indicate that the presence of the Arch transgene reduced Hcrt neuron excitability in an expressiondependent manner.

To determine whether these changes affected the response of Hcrt/orexin neurons to a known activator of these cells, we applied oxytocin (300 nM) to Hcrt/orexin neurons from orexin/Arch mice. In aLE mice (Fig. 3Gi), oxytocin significantly depolarized Hcrt/orexin neurons $(\Delta=4.52 \pm 1.11 \mathrm{mV}, n=7$; repeatedmeasures ANOVA, $\left.F_{(2,6)}=18.60, p=0.0006\right)$, evoking firing activity in some cells. This response was absent in aHE mice $(\Delta=$ $-0.24 \pm 0.53 \mathrm{mV}, n=8$; repeated-measures ANOVA, $F_{(2,7)}=$ $0.23, p=0.78$; Fig. 3Gii). Oxytocin (300 nM) significantly increased $V_{\mathrm{m}}$ in Hcrt/orexin neurons from aLE mice but not from aHE mice (Fig. 3Giii). These data indicate that high expression levels of the Arch transgene modify the electrical properties of Hcrt neurons independently from its opsin function, which may affect other behaviors that are dependent on Hcrt/orexin signaling.

\section{Orexin/Arch mice exhibit aberrant metabolic characteristics}

Hcrt/orexin neurons are known to exert effects on motor activity and metabolic parameters. As shown in Figure 3, multiple changes in electrophysiological characteristics of Hcrt/orexin neurons occur in 8-week-old orexin/Arch mice with high expression of the Arch transgene (aHE orexin/Arch mice). Consequently, we assessed whether these changes observed in vitro were reflected in changes in metabolism in vivo. Using the CLAMS, we measured $48 \mathrm{~h}$ FI, $x$ and $z$ activity, and RER in aLE and aHE orexin/Arch mice, ATAX mice, and WT mice during the light, dark, and $24 \mathrm{~h}$ periods (Fig. 4A). ATAX mice are an established model of Hcrt/orexin cell loss that mimic an NT1-like phenotype (Hara et al., 2001).

ATAX mice $(n=4)$ consumed significantly less food (Fig. $4 A i i$, top) than the other genotypes across the time periods analyzed (light: $0.07 \pm 0.008 \mathrm{~g} / \mathrm{h}$; dark: $0.12 \pm 0.004 \mathrm{~g} / \mathrm{h} ; 24 \mathrm{~h}: 0.09 \pm$ $0.006 \mathrm{~g} / \mathrm{h}$; two-way ANOVA: $F_{(3,15)}=7.59, p=0.003$ for time; $F_{(2,30)}=228.25, p<0.0001$ for genotype). During the light phase, aHE mice ate amounts comparable with ATAX mice (light: $0.08 \pm 0.008 \mathrm{~g} / \mathrm{h}$ vs WT light: $0.11 \pm 0.003 \mathrm{~g} / \mathrm{h}$ ) but less than either aLE or WT mice. 
A
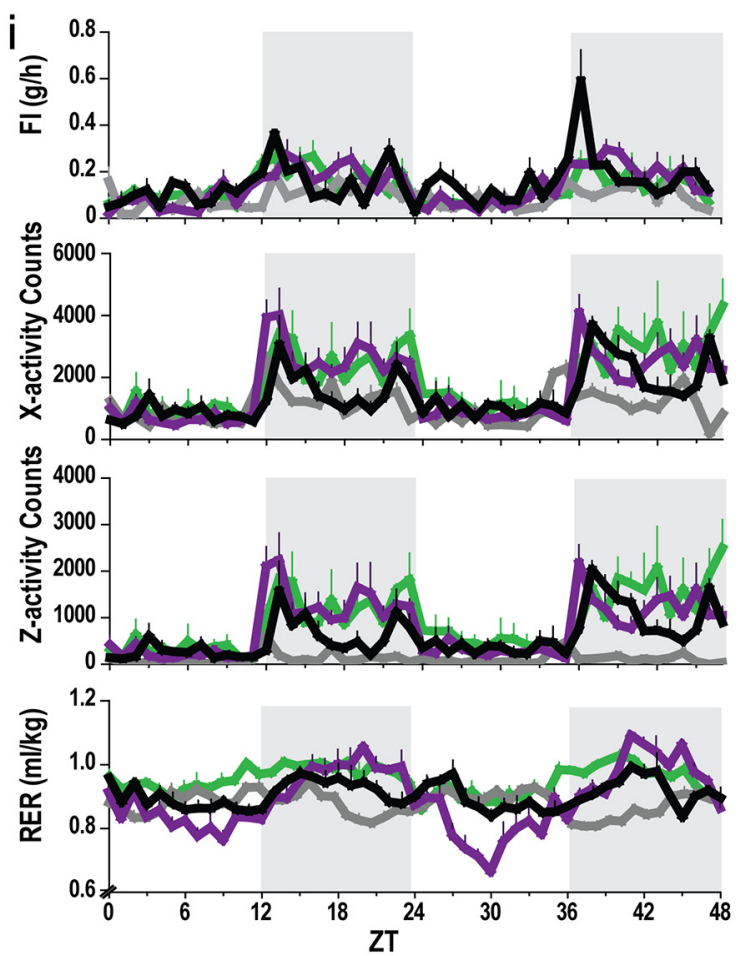

B

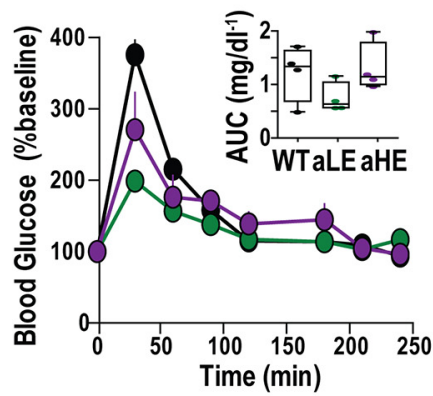

C

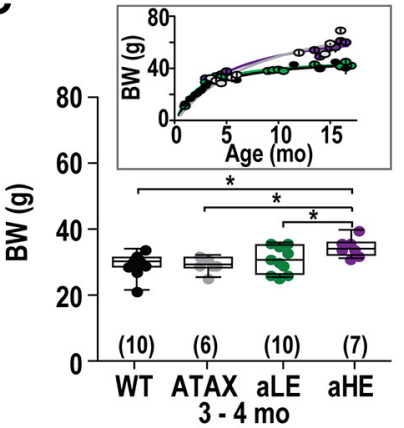

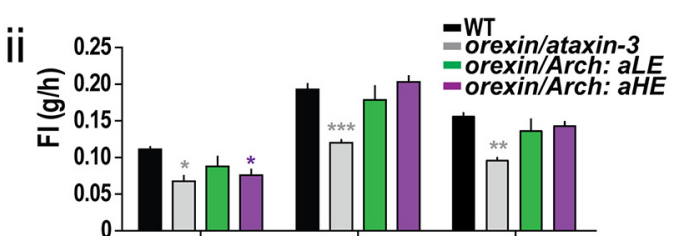
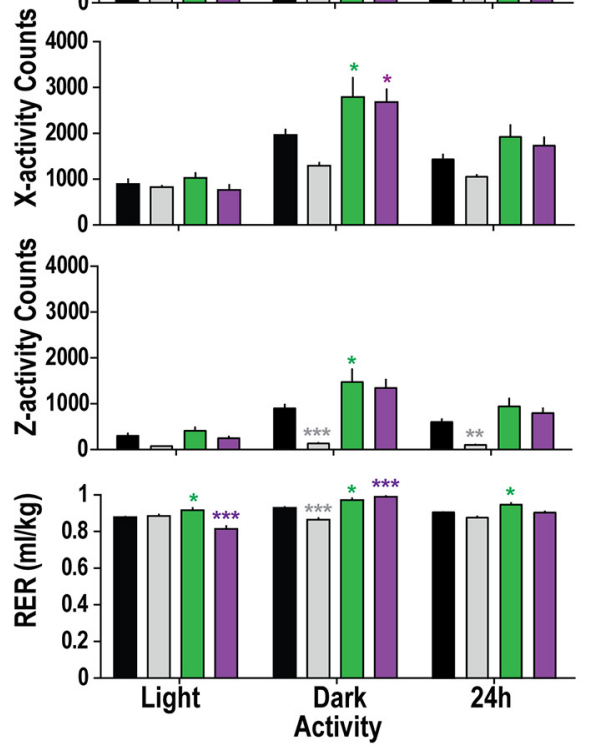

D

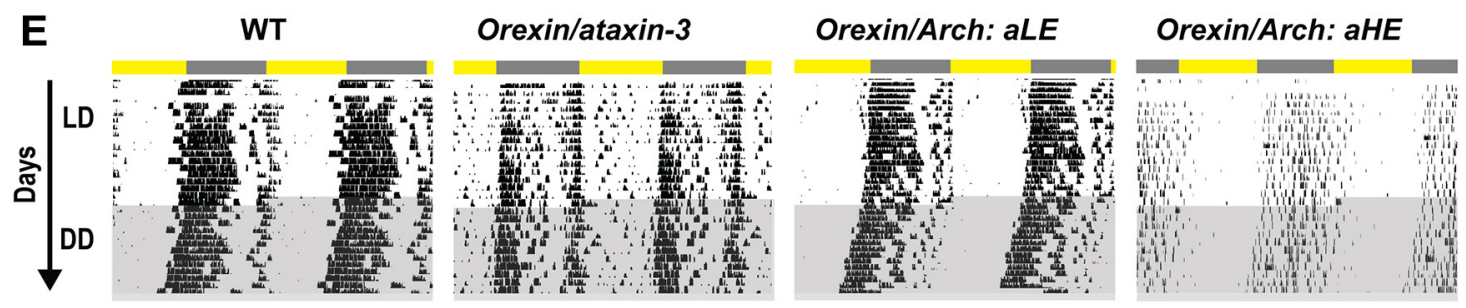

Figure 4. Aberrant circadian and metabolic features of orexin/Arch mice compared with orexin/Ataxin-3 (ATAX) and WT mice. A, Hourly metabolic rate over a $48 \mathrm{~h}$ period (Ai) and summed for the light, dark, and $24 \mathrm{~h}$ periods (Aii) revealed reduced Fl, $z$ activity, and RER during the dark phase in $A T A X$ mice and reduced $\mathrm{Fl}$ and RER in aHE mice during the light phase relative to other strains. Both aLE and aHE mice had greater $x$ activity and RER during the dark phase relative to other strains. $B$, By 12 weeks of age, the body weight of orexin/Arch aHE mice exceeded that of both WT and ATAX mice. Inset, Body weight trajectory with age across the different strains using a line of best fit for each genotype $\left(0.83>R^{2}>0.90\right)$. C, Age-matched mice were used to assess postmortem body composition; aHE orexin/Arch mice had significantly elevated adiposity, particularly increased epididymal and subcutaneous fat mass. $\boldsymbol{D}$, Male mice ( 9 weeks) were fasted overnight for a glucose tolerance test; no significant differences were observed across genotypes. $E$, Representative wheel-running actograms represent comparable locomotor rhythms between WT and aLE orexin/Arch mice under both LD (yellow bar: gray bar) and DD (gray shaded area) conditions. In contrast, aHE orexin/Arch mice have greatly reduced running wheel activity. Two-way ANOVA with Bonferroni post hoc test: ${ }^{*} p<0.05 ;{ }^{* *} p<0.01 ;{ }^{* * *} p<0.001$. Abd, Abdominal fat; EpD, epididymal fat pad; SubQ, subcutaneous fat pad.

$x$ and $z$ activity counts did not differ among genotypes across the $24 \mathrm{~h}$ period (Fig. 4 Aii). However, both orexin/Arch subgroups exhibited increased activity during the dark phase relative to WT mice ( $x$ activity: WT: $1963.35 \pm 118.6$ beam breaks, aLE: $2792.10 \pm 421.2$ beam breaks, aHE: $2681.65 \pm 280.68$ beam breaks; $p=0.01$ for genotype by two-way ANOVA).
Surprisingly, significant differences in RER occurred between WT mice and both subgroups of orexin/Arch mice (Fig. 4Aii, bottom). During the light phase, aHE mice had significantly lower RER than WT (aHE: $0.81 \pm 0.01$; WT: $0.88 \pm 0.003)$, but aLE mice had increased RER (aLE: $0.92 \pm 0.012 ; p=0.0004$ for genotype by two-way ANOVA). During the dark phase, both 
Table 2. Diurnal and circadian parameters of running wheel activity rhythms in WT, orexin/Ataxin-3, and orexin/Arch transgenic mice

\begin{tabular}{|c|c|c|c|c|c|c|c|}
\hline \multirow[b]{2}{*}{ Strain } & \multirow[b]{2}{*}{$N$} & \multicolumn{2}{|c|}{ Mean revolutions/min } & \multicolumn{2}{|l|}{ Tau (h) } & \multicolumn{2}{|l|}{ Amplitude } \\
\hline & & LD & $\mathrm{DD}$ & LD & $\mathrm{DD}$ & LD & $\mathrm{DD}$ \\
\hline WT & 8 & $8.56 \pm 2.59$ & $11.11 \pm 2.22$ & $24.02 \pm 0.03$ & $23.77 \pm 0.05$ & $2028 \pm 512$ & $992 \pm 188$ \\
\hline Orexin/Arch: aHE & 6 & $2.06 \pm 0.95^{\#}$ & $2.37 \pm 0.90^{* *, \#}$ & $24.00 \pm 0.03$ & $23.61 \pm 0.13^{t t t}$ & $1707 \pm 402$ & $1486 \pm 434$ \\
\hline 2-way ANOVA & & \multicolumn{2}{|c|}{$F_{(3,29)}=3.03 ; p=0.0452$} & \multicolumn{2}{|c|}{$F_{(3,29)}=3.42 ; p=0.03$} & \multicolumn{2}{|c|}{$F_{(3,29)}=2.18 ; p=0.112$} \\
\hline
\end{tabular}

${ }^{*} p<0.05 ;{ }^{* *} p<0.01 ;{ }^{* * *} p<0.001$ versus WT.

${ }^{\dagger} p<0.05 ;{ }^{{ }^{\dagger}} p<$ versus ATAX; ${ }^{+t+} p<0.001$ versus ATAX.

${ }^{\#} p<0.05 ;{ }^{\# \# \#} p<0.001$ versus alE.

${ }^{\wedge} p<0.05$ versus $a H E$.

Table 3. Daily food consumption and weekly increase in body weight in Orexin/Arch, Orexin/Ataxin-3, and WT mice

\begin{tabular}{|c|c|c|c|c|c|c|c|}
\hline \multirow[b]{2}{*}{ Strain } & \multirow[b]{2}{*}{$N$} & \multicolumn{3}{|c|}{ Normalized Fl (g/d) } & \multicolumn{3}{|c|}{ Body weight change (g/week) } \\
\hline & & LD & $\mathrm{DD}$ & LD (no wheels) & LD & $\mathrm{DD}$ & LD (no wheels) \\
\hline WT & 5 & $0.31 \pm 0.01$ & $0.33 \pm 0.02$ & $0.31 \pm 0.02$ & $-0.40 \pm 0.25$ & $0.53 \pm 0.17$ & $0.73 \pm 0.19$ \\
\hline Two-way ANOVA & & \multicolumn{3}{|c|}{$F_{(6,33)}=0.64, p=0.694$} & \multicolumn{3}{|c|}{$F_{(6,33)}=1.77, p=0.1363$} \\
\hline
\end{tabular}

subgroups of orexin/Arch mice had significantly higher RER values than WT mice. Together, these data indicate metabolic abnormalities in orexin/Arch mice, presumably due to an unexpected transgene effect on Hcrt/orexin neuronal function.

\section{Phenotypic abnormalities in aHE orexin/Arch mice}

As described above, data collected in the CLAMS instrument indicated aberrant physiology in orexin/Arch mice that was distinct from that of $A T A X$ mice. To more thoroughly investigate energy metabolism, we assessed growth rate, body composition, glucose tolerance, and circadian control of locomotor activity.

Body weight varied significantly across genotypes with age (Fig. 4B). At 3-4 months, the body weight of aHE mice (35.71 \pm $1.13 \mathrm{~g}, n=7$ ) exceeded that of all other groups (WT: $30.37 \pm$ $1.11 \mathrm{~g}, n=10$; aLE: $31.70 \pm 1.33 \mathrm{~g}, n=10$; ATAX: $30.17 \pm 0.89 \mathrm{~g}$, $n=6$; one-way ANOVA, $\left.F_{(3,29)}=3.93, p=0.02\right)$. By $14-16$ months (Fig. $4 B$, inset), both aHE and ATAX mice were obese relative to either WT or aLE mice but were not significantly different from each other (WT: $39.80 \pm 2.15 \mathrm{~g}, n=5$; aLE: $42.33 \pm$ $0.98 \mathrm{~g}, n=6$; aHE: $56.80 \pm 1.79 \mathrm{~g}, n=10$; ATAX: $55.20 \pm 4.03 \mathrm{~g}$, $n=5$; one-way ANOVA, $\left.F_{(3,22)}=14.68, p<0.0001\right)$.

Since body weight was altered in aHE mice and ATAX mice, we assessed whether this was due to increased fat mass. Mice from all groups were killed at various ages (Fig. $4 C$, inset) and the fat pads weighed (Fig. 4C). We found that epididymal and subcutaneous fat mass was comparable between ATAX $(n=6)$ and aHE mice $(n=14)$. The increase in both aHE fat pads was significantly different from WT ( $n=10$; two-way ANOVA, $p<0.05$ for epididymal and subcutaneous).

We also assessed glucose tolerance in mice before age-related fat mass changes to determine whether fasting glucose levels predicted increased body weight in later life. A glucose tolerance test performed after overnight fasting did not reveal any significant differences in glucose tolerance among the strains examined (Fig. $4 D$; one-way ANOVA, $\left.F_{(2,9)}=1.96, p=0.19\right)$.

Since the circadian clock has been linked to metabolic regulation, we measured wheel-running activity under LD and DD conditions as an index of clock function in adult mice. Mice were acclimated to running wheels for $5 \mathrm{~d}$ before assessing voluntary measures of circadian locomotor activity (Fig. 4E; Table 2). Although mice of all strains entrained to an LD cycle, there were clear differences in the amount of running wheel activity across strains (two-way ANOVA, $F_{(3,29)}=3.03, p=0.004$ ) with aHE mice being the least active. The reduced activity in both aHE and $A T A X$ mice was significant under DD (two-way ANOVA, $p=$ 0.045; Bonferroni post hoc test, $p<0.01$ for aHE vs WT and $p<$ 0.05 for $A T A X$ vs WT). In DD conditions, the endogenous period of ATAX mice remained close to $24 \mathrm{~h}$ and was thus significantly longer than all other genotypes $(p<0.001)$. There was no significant genotype $\times$ time interaction for circadian amplitude (twoway ANOVA, $\left.F_{(3,29)}=2.18 ; p<0.11\right)$.

FI and body weight were also measured in mice that had access to running wheels. FI was normalized to the body weight of each individual mouse (Table 3). Although no significant differences were found, there was a trend toward increased body weight gain in aHE mice during DD compared with WT.

There was some variation in age (WT: $6.3 \pm 0.7$ months, $n=$ 7; aLE: $7.3 \pm 1.2$ months, $n=11$; aHE: $9.1 \pm 2.2$ months, $n=6$; ATAX: $4.8 \pm 0.3$ months, $n=8$ ) and body weight (WT $=37.2 \pm$ $2.6 \mathrm{~g}$, aLE: $35.7 \pm 1.6 \mathrm{~g}$, aHE: $47.6 \pm 5.8 \mathrm{~g}, A T A X: 33.1 \pm 1.2 \mathrm{~g}$ ) across strains of mice given access to running wheels. Since these differences in age may have contributed to the differences in wheel-running activity recorded, we removed the oldest aHE mice from the analysis, which reduced the mean age of aHE mice to $5.5 \pm 2.5$ months $(n=4)$. Nevertheless, the reduced wheelrunning of aHE mice in DD compared with WT persisted (aHE: $3.15 \pm 1.15$ revolutions/min vs WT: $11.90 \pm 2.42$ revolutions/ min; one-way ANOVA, $p=0.04$; data not shown).

\section{$A T A X$ and Orexin/Arch transgenic mice exhibit distinct differences in sleep-wake architecture and EEG spectra}

The sleep-wake patterns and EEG spectral features of aLE and aHE orexin/Arch mice were compared with WT and with ATAX mice, an established model of Hcrt/orexin cell loss that results in NT1. Baseline recordings of sleep-wake suggested little difference among strains in the hourly amounts of wakefulness (Fig. 5Ai, left), but two-way repeated-measures ANOVA revealed a nominally significant genotype $X$ time interaction when the data were analyzed across the light and dark phases and the entire $24 \mathrm{~h}$ period $\left(F_{(6,48)}=2.9 ; p=0.017\right.$; Bonferroni-corrected threshold $\alpha=0.006$; Table 4). Wake bout architecture (Fig. 5Ai, right) differed among strains with significant genotype $\times$ time interac- 
A
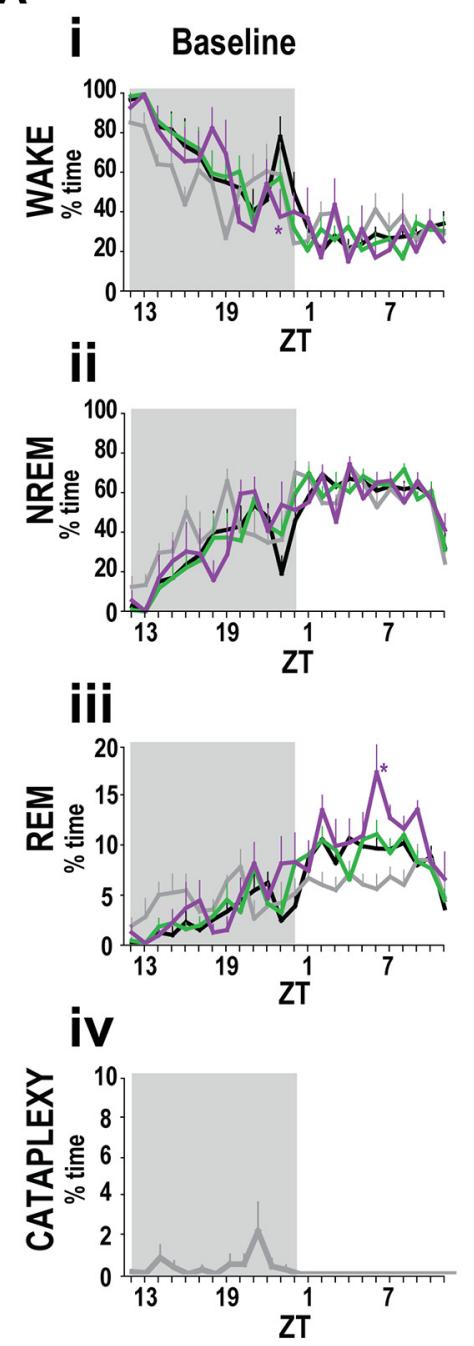

C
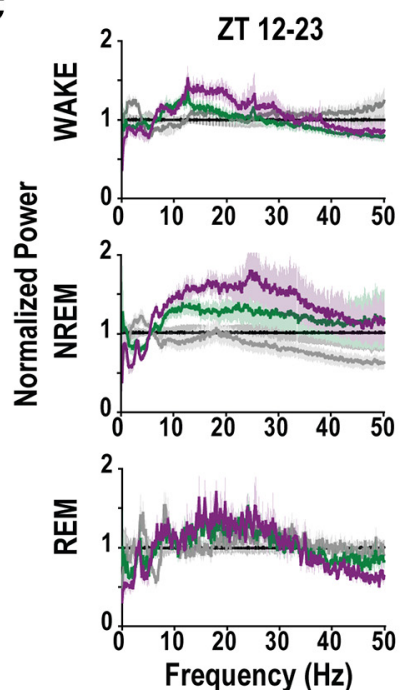
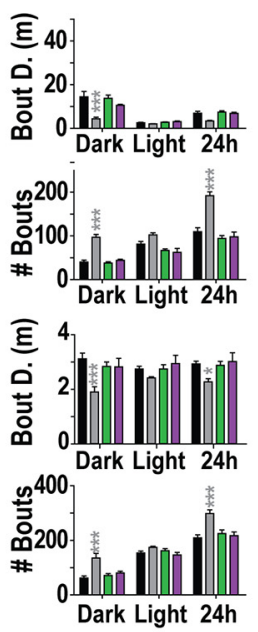

巨ิ 4

○े 2.
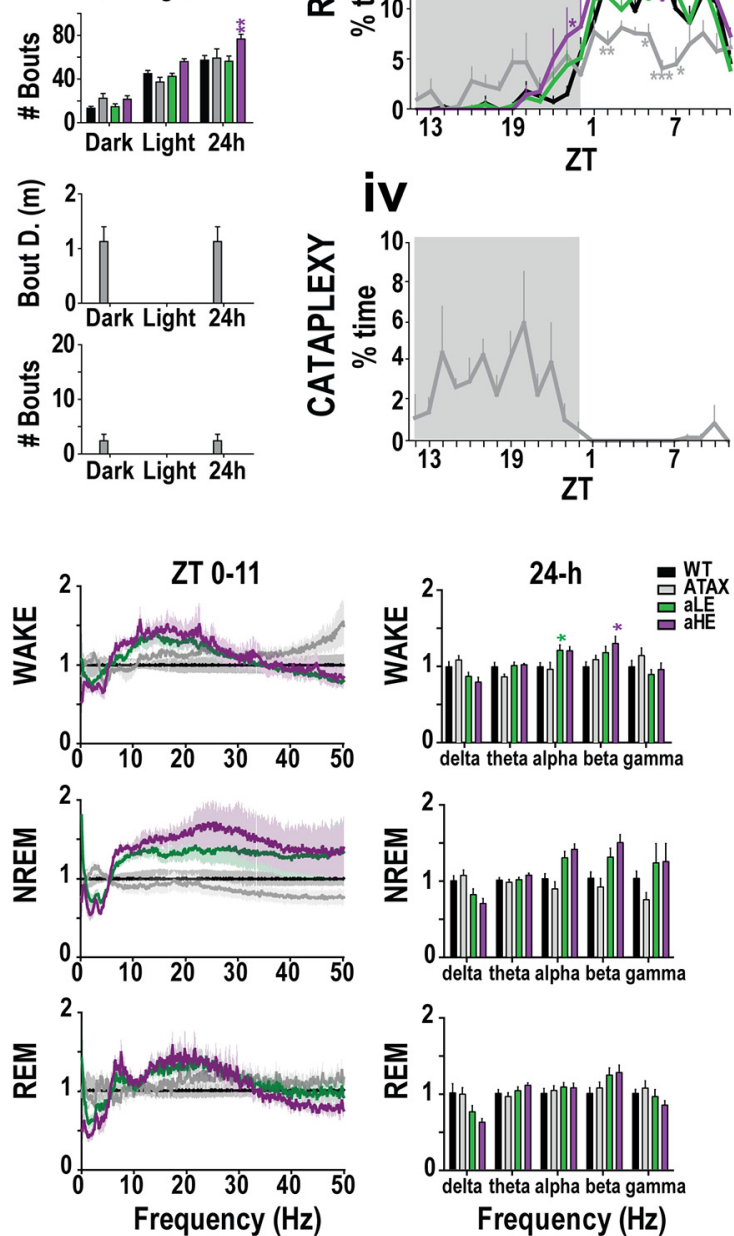

B
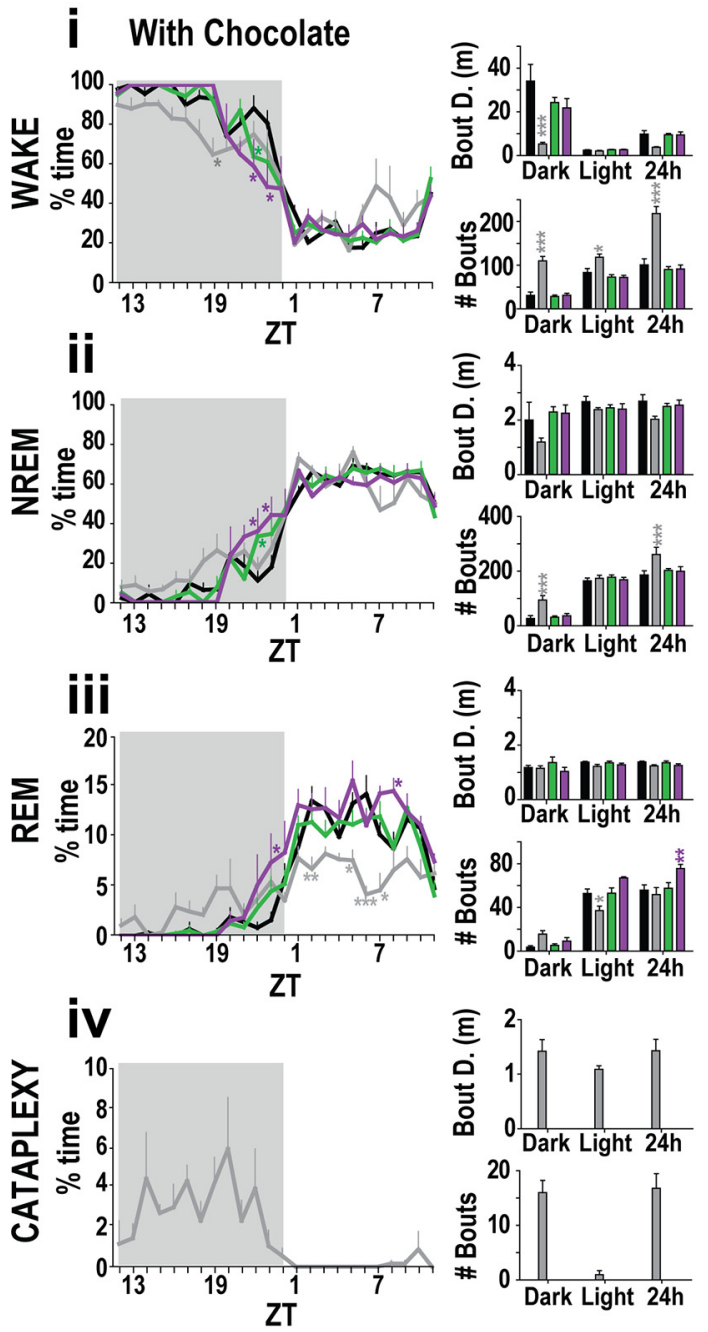

Figure 5. EEG phenotyping of male orexin/Ataxin-3 (ATAX) and orexin/Arch mice reveals distinct differences in sleep-wake architecture and EEG spectral composition. $\boldsymbol{A}$, Diurnal distribution of wakefulness (Ai) and NREM sleep (Aii) in both aLE and aHE orexin/Arch mice is comparable with age-matched male WT mice but distinct from that of $A T A X$ mice. However, the number of REM bouts in aHE orexin/Arch mice was significantly increased over the $24 \mathrm{~h}$ period relative to the other strains (Aiii). Spontaneous cataplexy was only observed in ATAX mice (Aiv). Repeated-measures ANOVA revealed strain differences in hourly LMA (Fig. 5-1 Ai, available at https://doi.org/10.1523/JNEUROSCI.0311-19.2019.f5-1) without a significant effect on T (Fig. 5-1 Aii, available at https://doi.org/ 10.1523/JNEUROSCI.0311-19.2019.f5-1). B, The chocolate-cataplexy assay was used to determine whether cataplexy could be elicited in orexin/Arch mice under rewarding conditions. Presentation of chocolate at ZT12 significantly increased time spent awake (Bi) and reduced NREM sleep (Bii) during the dark phase for all strains. The percent time in REM sleep (Figure legend continues.) 
Table 4. Basal sleep/wake parameters of WT, orexin/Ataxin-3, and orexin/Arch transgenic mice

\begin{tabular}{|c|c|c|c|c|c|c|c|c|c|c|}
\hline \multirow[b]{2}{*}{ Strain } & \multirow[b]{2}{*}{$N$} & \multicolumn{3}{|l|}{ Percentage of time } & \multicolumn{3}{|l|}{ No. of bouts } & \multicolumn{3}{|c|}{ Mean bout duration (min) } \\
\hline & & Light phase & Dark phase & $24 \mathrm{~h}$ & Light phase & Dark phase & $24 \mathrm{~h}$ & Light phase & Dark phase & $24 \mathrm{~h}$ \\
\hline \multicolumn{11}{|l|}{ Wake } \\
\hline WT & 8 & $29.91 \pm 0.96$ & $71.08 \pm 3.35$ & $50.49 \pm 1.81$ & $81.12 \pm 6.22$ & $40.25 \pm 4.38$ & $109.75 \pm 9.04$ & $2.59 \pm 0.19$ & $14.24 \pm 2.45$ & $6.96 \pm 0.78$ \\
\hline Orexin/Ataxin-3 & 5 & $31.91 \pm 0.93$ & $59.08 \pm 4.91$ & $45.14 \pm 2.08$ & $102.40 \pm 4.54^{*}$ & $97.00 \pm 6.46^{* * *}$ & $192.00 \pm 9.04^{* * *}$ & $2.09 \pm 0.11$ & $4.45 \pm 0.68^{* * *}$ & $3.34 \pm 0.34$ \\
\hline Orexin/Arch:alE & 10 & $27.14 \pm 1.16$ & $69.49 \pm 2.62$ & $48.31 \pm 1.34$ & $66.50 \pm 4.06^{t+t}$ & $38.10 \pm 2.77^{\mathrm{ttt}}$ & $94.50 \pm 6.33^{\mathrm{ttt}}$ & $2.76 \pm 0.17$ & $13.83 \pm 1.46^{t+t}$ & $7.43 \pm 0.59^{\dagger}$ \\
\hline Orexin/Arch:aHE & 5 & $27.85 \pm 1.69$ & $65.51 \pm 1.88$ & $46.68 \pm 1.68$ & $62.40 \pm 9.06^{t \dagger}$ & $44.20 \pm 2.63^{t+t}$ & $98.00 \pm 11.06^{t+t}$ & $3.11 \pm 0.24$ & $10.59 \pm 0.41^{\dagger+}$ & $6.86 \pm 0.50$ \\
\hline Two-way RM ANOVA & & \multicolumn{3}{|c|}{$F_{(6,48)}=2.9 ; p=0.017$} & \multicolumn{3}{|c|}{$F_{(6,48)}=14.4 ; p<0.0001$} & \multicolumn{3}{|c|}{$F_{(6,48)}=5.42 ; p=0.0002$} \\
\hline \multicolumn{11}{|l|}{ NREM sleep } \\
\hline WT & 8 & $58.79 \pm 1.25$ & $26.64 \pm 3.03$ & $42.72 \pm 1.94$ & $153.75 \pm 7.46$ & $62.25 \pm 7.28$ & $209.00 \pm 11.12$ & $2.77 \pm 0.10$ & $3.14 \pm 0.21$ & $2.95 \pm 0.10$ \\
\hline Orexin/Ataxin-3 & 5 & $59.06 \pm 1.11$ & $36.24 \pm 4.70$ & $47.65 \pm 2.44$ & $174.20 \pm 7.88$ & $135.60 \pm 16.53^{* * *}$ & $298.60 \pm 13.24^{* * *}$ & $2.44 \pm 0.05$ & $1.92 \pm 0.19$ & $2.29 \pm 0.11$ \\
\hline Orexin/Arch:aLE & 10 & $61.31 \pm 1.71$ & $27.81 \pm 2.20$ & $44.56 \pm 1.37$ & $162.00 \pm 7.88$ & $71.50 \pm 7.00^{\mathrm{tt} t}$ & $225.10 \pm 12.99^{\mathrm{ttt}}$ & $2.77 \pm 0.15$ & $2.86 \pm 0.16$ & $2.90 \pm 0.15$ \\
\hline Orexin/Arch:aHE & 5 & $59.08 \pm 2.77$ & $31.16 \pm 1.83$ & $45.11 \pm 2.23$ & $146.00 \pm 9.79$ & $80.60 \pm 6.17^{\dagger}$ & $217.40 \pm 13.51^{\mathrm{ttt}}$ & $2.97 \pm 0.30$ & $2.84 \pm 0.32$ & $3.05 \pm 0.33$ \\
\hline Two-way RM ANOVA & & \multicolumn{3}{|c|}{$F_{(6,48)}=2.21 ; p=0.06$} & \multicolumn{3}{|c|}{$F_{(6,48)}=5.36 ; p=0.0003$} & \multicolumn{3}{|c|}{$F_{(6,48)}=3.02 ; p=0.014$} \\
\hline \multicolumn{11}{|l|}{ REM sleep } \\
\hline WT & 8 & $8.49 \pm 0.64$ & $2.27 \pm 0.32$ & $5.38 \pm 0.40$ & $45.00 \pm 2.96$ & $13.25 \pm 1.77$ & $57.37 \pm 4.23$ & $1.32 \pm 0.05$ & $1.22 \pm 0.06$ & $1.33 \pm 0.04$ \\
\hline Orexin/Ataxin-3 & 5 & $6.30 \pm 0.45^{*}$ & $4.24 \pm 0.62$ & $5.27 \pm 0.51$ & $37.20 \pm 4.40$ & $22.40 \pm 4.40$ & $59.20 \pm 8.54$ & $1.24 \pm 0.07$ & $1.42 \pm 0.11$ & $1.31 \pm 0.06$ \\
\hline Orexin/Arch:alE & 10 & $8.77 \pm 0.51^{\mathrm{tt}, \wedge}$ & $2.70 \pm 0.48$ & $5.73 \pm 0.43$ & $42.50 \pm 2.81$ & $14.70 \pm 2.65$ & $56.40 \pm 4.74^{\wedge}$ & $1.50 \pm 0.08$ & $1.31 \pm 0.08$ & $1.48 \pm 0.08$ \\
\hline Orexin/Arch:aHE & 5 & $10.80 \pm 0.98 *, t+\dagger$ & $3.33 \pm 0.23$ & $7.06 \pm 0.43$ & $56.00 \pm 3.17^{\dagger}$ & $21.60 \pm 3.17$ & $76.60 \pm 4.50^{* *}$ & $1.37 \pm 0.10$ & $1.14 \pm 0.12$ & $1.31 \pm 0.07$ \\
\hline Two-way ANOVA & & \multicolumn{3}{|c|}{$F_{(6,48)}=9.69 ; p<0.0001$} & \multicolumn{3}{|c|}{$F_{(6,48)}=4.42 ; p=0.0012$} & \multicolumn{3}{|c|}{$F_{(6,48)}=1.82 ; p=0.114$} \\
\hline Cataplexy & & & & & & & & & & \\
\hline Orexin/Ataxin-3 & 5 & $0.00 \pm 0.00$ & $0.44 \pm 0.25$ & $0.22 \pm 0.12$ & $0.00 \pm 0.00$ & $2.40 \pm 1.21$ & $2.40 \pm 1.21$ & - & $1.13 \pm 0.27$ & $1.13 \pm 0.27$ \\
\hline
\end{tabular}

tions for both the number of wake bouts $\left(F_{(6,48)}=14.4 ; p<\right.$ $0.0001)$ and wake bout duration $\left(F_{(6,48)}=5.42 ; p=0.0002\right)$. Post $h o c$ analyses revealed that ATAX mice were the source of this variation, exhibiting more wake bouts $(p<0.001)$ of shorter duration $(p<0.001)$ during the dark phase compared with WT mice.

Like wakefulness, the hourly percentage of NREM sleep did not differ across strains (Fig. 5Aii) but, when examined across the light and dark phases and the entire $24 \mathrm{~h}$ period, two-way repeated-measures ANOVA revealed significant genotype $\times$ time interactions (Table 4) for the number of NREM bouts $\left(F_{(6,48)}=5.36 ; p=0.0003\right)$ and nominally significant NREM bout duration $\left(F_{(6,48)}=3.02 ; p=0.014\right.$; Bonferroni-corrected threshold $\alpha=0.006)$. As during wakefulness, these differences were due to the ATAX mice, which had more than twice as many NREM bouts during the dark period compared with WT mice $(p<0.001$; Fig. 5Aii, right)

In contrast to wake and NREM sleep, two-way repeatedmeasures ANOVA revealed a significant genotype $X$ time interaction in the hourly amount of REM sleep $\left(F_{(69,552)}=2.02 ; p<\right.$ 0.0001; Fig. 5Aiii). Further analysis indicated that ATAX mice had less REM sleep than WT during the $12 \mathrm{~h}$ light phase $(p<$ $0.05)$, whereas aHE mice had more REM during this phase $(p<$

\footnotetext{
$\leftarrow$

(Figure legend continued.) also decreased, except in ATAX mice (Biii). Chocolate also robustly increased cataplexy in ATAX mice but did not elicit cataplexy in orexin/Arch mice (Biv). Neither LMA $(p=0.12$; Fig. 5-1Bi, available at https://doi.org/10.1523/JNEUROSCI.031119.2019.55-1) nor $\mathrm{T}_{\mathrm{b}}(p=0.21$; Fig. 5-1Bii, available at https://doi.org/10.1523/ JNEUROSCI.0311-19.2019.f5-1) was significantly different among genotypes in the CCA. C, Spectral analysis of normalized EEG power across the active phase (ZT12-ZT23) and inactive phase (ZT24-ZT11) indicated significant changes in different bands across sleep-wake states in orexin/Arch mice during baseline recordings. During the $4 \mathrm{~h} \mathrm{SD}$, neither LMA ( $p=0.87$; Fig. 5-1Ci, available at https://doi.org/10.1523/JNEUROSCI.0311-19.2019.f5-1) nor $\mathrm{T}_{\mathrm{b}}(p=0.089$; Fig. 5-1Cii, available at https://doi.org/10.1523/JNEUROSCI.0311-19.2019.f5-1) differed across strains. Bout D., Bout duration. Two-way ANOVA with Bonferroni post hoc test: ${ }^{*} p<0.05$; ${ }^{* *} p<0.01 ;{ }^{* * *} p<0.001$ relative to WT.
}

$0.01)$ and more REM bouts across the entire $24 \mathrm{~h}$ period than WT mice $(p<0.01$; Table 4$)$.

Analysis of hourly LMA revealed a genotype $\times$ time interaction (two-way repeated-measures ANOVA, $F_{(69,552)}=1.33 ; p=$ 0.04; Fig. 5-1Ai, left, available at https://doi.org/10.1523/ JNEUROSCI.0311-19.2019.f5-1) that may reflect, in part, the tendency for older ATAX and aHE mice to exhibit reduced wheel-running activity compared with WTs (Fig. 4E). When the LMA data were binned into respective LD phases, however, the genotype $\times$ time of day interaction was not significant (two-way repeated-measures ANOVA, $F_{(6,48)}=1.80 ; p=0.12$; Fig. 5- $1 A i$, right, available at https://doi.org/10.1523/JNEUROSCI.031119.2019.f5-1). Basal $\mathrm{T}_{\mathrm{b}}$ was similar across strains when viewed either hourly (two-way repeated-measures ANOVA, $F_{(69,552)}=$ $1.13 ; p=0.24)$ or when binned into the light and dark phases (two-way repeated-measures ANOVA, $F_{(6,48)}=0.36 ; p=0.90$; Fig. 5-1Aii, available at https://doi.org/10.1523/JNEUROSCI. 0311-19.2019.f5-1).

Spontaneous cataplexy occurred exclusively in ATAX mice and was only observed during the dark phase (Fig. 5Aiv). Since Hcrt/orexin neurons appear to be hypofunctional in aHE orexin/ Arch mice, we used the CCA (Oishi et al., 2013) to determine whether we could elicit cataplectic attacks in aHE mice. Under the rewarding condition of the CCA, cataplexy increased substantially in ATAX mice (Fig. 5Biv vs Fig. 5Aiv) and was even observed during the light phase but remained restricted to this strain. Access to chocolate at the beginning of the dark phase prolonged wakefulness (Fig. $5 B i$ vs Fig. 5Ai) and decreased NREM sleep during the dark phase (Fig. 5Bii vs Fig. 5Aii) in all genotypes. Chocolate availability at the beginning of the dark phase also tended to displace REM sleep into the light phase (Fig. 5Biii vs Fig. 5Aiii). LMA did not differ among genotypes in the CCA (Fig. 5-1Bi, available at https://doi.org/10.1523/ JNEUROSCI.0311-19.2019.f5-1) nor did $\mathrm{T}_{\mathrm{b}}$ (Fig. 5-1 Bii, available at https://doi.org/10.1523/JNEUROSCI.0311-19.2019.f5-1). LMA increased significantly during the CCA relative to baseline only in aLE mice (two-way repeated-measures ANOVA, 
A
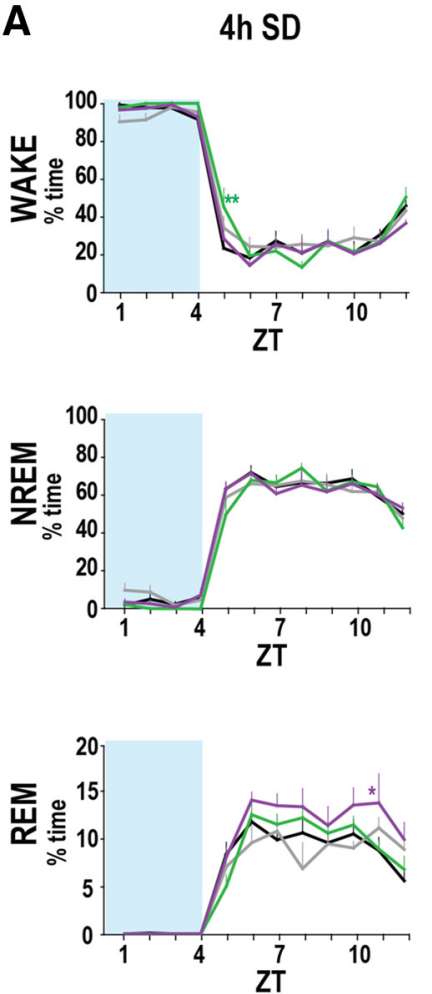
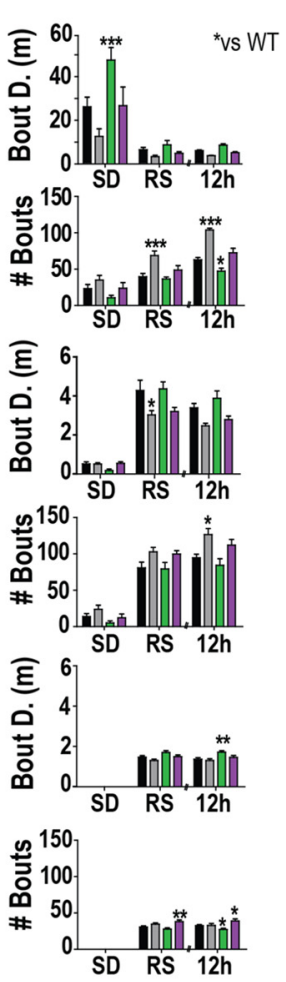

D

mMSLT
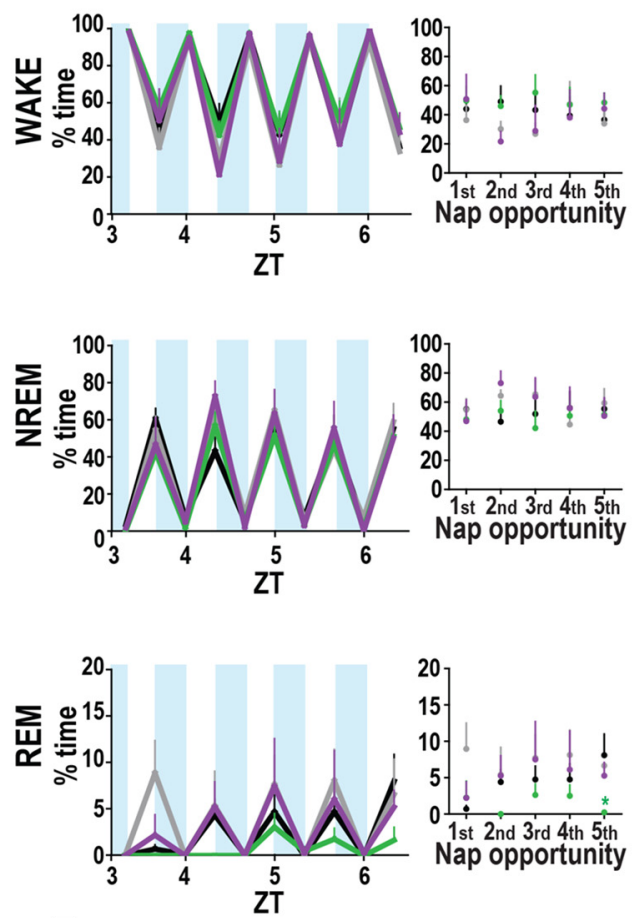

E
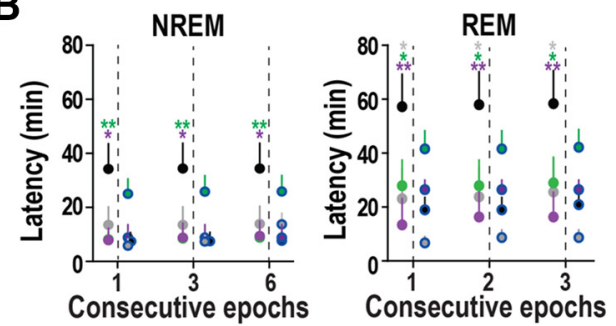

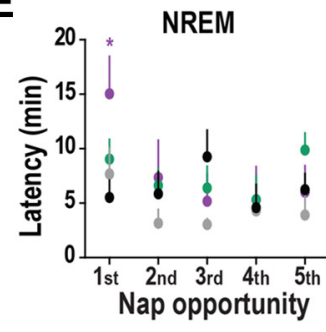

C

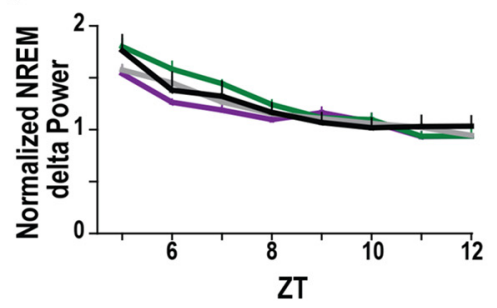

$\mathbf{F}$

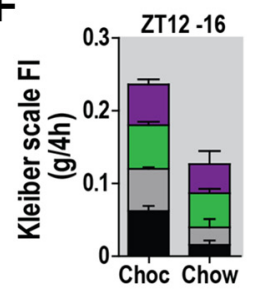

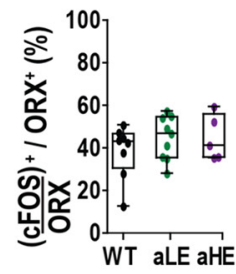

Figure 6. Sleep homeostasis parameters are normal in orexin/Arch mice. $A$, Percentage of wake, NREM, and REM sleep during a $4 \mathrm{~h}$ SD period initiated at ZTO (blue shading) and the subsequent recovery period from ZT4 to ZT12. B. The latencies to NREM and REM sleep were significantly shorter in orexin/Arch mice under baseline conditions, but all genotypes had similar latencies after $4 \mathrm{~h}$ SD. C, After 4hSD, all mice increased EEG delta power during NREM sleep, indicating comparable sleep homeostasis across genotypes. D, An mMSLT, comprised of five 20 min SD sessions interspersed with five 20 min "nap" opportunities, was initiated at ZT3 to assess sleepiness of each genotype. $E$, Latencies to NREM and REM sleep for all 4 genotypes during each "nap" opportunity of the mMSLT indicated that ATAX mice had significantly shorter REM latencies during the third and fourth nap opportunities. $F$, After initiation of access to chocolate at ZT12, total FI (i.e., consumption of chocolate and chow) was measured over the subsequent $4 \mathrm{~h}$. Orexin/Arch mice had food preference and consumption levels comparable with WT and ATAX mice. Mice were then killed at ZT16, and assessment of c-FOS expression in Hcrt/orexin neurons indicated that orexin/Arch mice had levels of CFOS in Hcrt/orexin neurons comparable with WT mice, and that cFOS expression was not specific to Hcrt/orexin neurons within the LHA. Values are mean \pm SEM. ${ }^{*} p<0.05,{ }^{* *} p<0.01,{ }^{* * *} p<0.001$ as in legends to Figures 4 and 5.

treatment $\times$ time interaction: $F_{(2,18)}=42.13 ; p<0.0001$; Fig. 5-1Bi, available at https://doi.org/10.1523/JNEUROSCI.031119.2019.f5-1 vs Fig. 5-1 Ai, available at https://doi.org/10.1523/ JNEUROSCI.0311-19.2019.f5-1). In comparison, $\mathrm{T}_{\mathrm{b}}$ increased during the CCA in all phases (Fig. 5-1 Bii, available at https:// doi.org/10.1523/JNEUROSCI.0311-19.2019.f5-1) with significant treatment $\times$ time interactions for WT (two-way repeatedmeasures ANOVA, $F_{(2,14)}=14.29 ; p<0.0001$ ), aLE (two-way repeated-measures ANOVA, $\left.F_{(2,18)}=18.79 ; p<0.0001\right)$, and
aHE (two-way repeated-measures ANOVA, $F_{(2,8)}=14.88 ; p=$ $0.0020)$ mice.

Spectral analysis of baseline wake, NREM, and REM states during the active (ZT12-ZT23) and inactive phases (ZT0-ZT11) revealed nominally significant (Bonferroni-corrected threshold $\alpha=0.02$ ) differences in the frequency bands in orexin/Arch mice (Fig. 5C), despite relatively normal sleep-wake architecture. These differences were more prominent in total spectral power during wake across the $24 \mathrm{~h}$ baseline period (two-way repeated- 
measures ANOVA, significant genotype $\times$ spectral band interactions for wake: $\left.F_{(12,96)}=3.05 ; p=0.001\right)$. There were also significant genotype $\times$ spectral band interactions for NREM $\left(F_{(12,96)}=2.35 ; p=0.01\right)$, whereas REM genotype $\times$ spectral band interaction did not reach significance $\left(F_{(12,96)}=0.027\right)$. Compared with WT mice in the wake state, aLE mice had increased alpha power and aHE had increased beta power. There was a trend for increased beta power in aHE during NREM and delta power in REM compared with WT (both $p=0.03$ ). In contrast, ATAX mice did not differ from WT mice in any EEG spectral parameter during either phase or across the $24 \mathrm{~h}$ period.

\section{Sleep homeostasis is intact in orexin/Arch transgenic mice}

Homeostatic sleep regulation was evaluated by analyzing the response to $4 \mathrm{~h}$ SD initiated at ZT0 (Fig. 6A). During the $4 \mathrm{~h} \mathrm{SD}$, all mice exhibited increased activity (Fig. 5-1Ci, available at https:// doi.org/10.1523/JNEUROSCI.0311-19.2019.f5-1). A genotype $\times$ time interaction was evident in the hourly $\mathrm{T}_{\mathrm{b}}$ data (two-way repeated-measures ANOVA, $F_{(69,552)}=1.40 ; p=0.02$; Fig. 5-1Cii, left, available at https://doi.org/10.1523/JNEUROSCI. 0311-19.2019.f5-1) that was not significant when the data were collapsed into bins corresponding to the light and dark phases (two-way repeated-measures ANOVA, $F_{(6,48)}=1.98 ; p=0.089$; Fig. 5-1Cii, right, available at https://doi.org/10.1523/JNEUROSCI. 0311-19.2019.f5-1).

$A T A X$ mice were the most difficult of the 4 strains to keep awake across the $4 \mathrm{~h}$ SD period (Fig. $6 A$ ). During the recovery sleep opportunity period (ZT5-ZT12), all genotypes exhibited a similar distribution of sleep-wake. Under baseline conditions, the latency to NREM (measured from light onset) was consistently shorter in orexin/Arch mice compared with WTs (Fig. 6B), and both orexin/Arch and ATAX mice had significantly faster REM sleep onsets than WT mice. Following SD, all genotypes had a reduced NREM and REM sleep latency (measured from cessation of SD) that did not differ across strains. NRD was elevated during recovery sleep in all strains (Fig. 6C), suggesting that the sleep homeostat is unaltered in either orexin/Arch or ATAX mice.

To assess sleep propensity, we used an mMSLT that began at ZT3 to further challenge the sleep homeostat (Fig. 6D). We found no significant difference across genotypes in wake or NREM states during the five 20 min nap opportunities. NREM latency was comparable across genotypes, except for the first nap opportunity, during which aHE mice took longer to enter NREM (Fig. 6E). REM sleep latencies were comparable between WT and orexin/Arch mice, but ATAX mice tended to have shorter latencies, which were significant in nap opportunities 3 and 4 (Fig. 6E).

\section{Food preference and c-FOS expression across genotypes}

To determine whether aberrant Hcrt/orexin neuron function affected food preference between chow and chocolate, we performed a final CCA in which mice were killed $4 \mathrm{~h}$ after chocolate presentation (Oishi et al., 2013). We found that all genotypes preferentially ate chocolate over chow (Fig. $6 F$ ) and only aLE mice ate significantly more chow than WT (two-way ANOVA, $p=0.03$ ). Overall, the genotypes exhibited a similar range of total FI and did not significantly differ from each other.

After $4 \mathrm{~h}$ exposure to chocolate from ZT12-ZT16, we assessed cellular activation in the LHA by measuring cFOS- and orexinIR. The number of Hcrt/orexin neurons counted was similar across WT and orexin/Arch mice (WT: $90.23 \pm 9.68$ cells; aLE: $83.93 \pm 1.04$ cells; aHE: $83.06 \pm 3.63$ cells) and largely absent in the mouse model of NT1 (ATAX: $2.5 \pm 0.51$ cells). The percentage of Hcrt/orexin neurons expressing c-FOS was comparable between WT $(38.48 \pm 4.4 \%, n=8)$ and orexin/Arch mice (aLE: $44.77 \pm 3.4 \%, n=10$; aHE: $44.90 \pm 4.7 \%, n=5)$. Total cFOS-IR indicated a more general activation of LHA neurons since the percentage of orexin ${ }^{+} / \mathrm{Fos}^{+}$neurons only accounted for a proportion of the total c-FOS-expressing cells in the LHA (WT: $44.63 \pm 4.18 \%$, aLE: $44.91 \pm 2.45 \%$, aHE: $49.22 \pm 4.49 \%$ ).

\section{Discussion}

Optogenetics has been a useful tool to further our understanding of the roles of different neurotransmitter systems in sleep-wake control (Jego et al., 2013; Konadhode et al., 2013; Irmak and de Lecea, 2014; Tsunematsu et al., 2014; Van Dort et al., 2015; Xu et al., 2015), including Hcrt/orexin (Adamantidis et al., 2007; Carter et al., 2009, 2012; Tsunematsu et al., 2011, 2013). In the present study, we used the light-sensitive Arch protein, which inhibits neurons through a proton pump. We found that photoillumination of Hcrt/orexin cells expressing Arch in transgenic orexin/Arch mice could induce sleep with short latency but that the efficacy of sleep induction was dependent on time of day and the degree of transgene expression. Furthermore, high Arch expression levels affected Hcrt neuron excitability and physiology, including energy metabolism, locomotor activity, REM sleep amount, and EEG spectra.

\section{Photoillumination-induced sleep in Orexin/Arch mice}

In studies in which the light-sensitive chloride channel halorhodopsin (Tsunematsu et al., 2011) or the proton pump ArchT (Tsunematsu et al., 2013) was expressed in Hcrt/orexin neurons, brief ( $1 \mathrm{~min}$ ) photoillumination induced NREM sleep with short latency only at certain times of day. Here, 1 min photoillumination of Arch-expressing Hcrt neurons induced NREM only during the light phase (Fig. 2C), supporting the concept of the Hcrt system as a "wake-maintaining" rather than a "wake-promoting" system. Narcoleptic patients and their animal counterparts that lack Hcrt neurons or have compromised Hcrt signaling are able to awaken but are less able to sustain long periods of wakefulness. Photic inhibition of Arch-containing Hcrt neurons during the light phase reduces Hcrt neural activity and creates conditions that are permissive for sleep to occur when sleep drive is high, resulting in shorter sleep onset latency. Conversely, 1 min photoillumination during the dark phase was ineffective because other arousal systems are activated at that time (Suzuki et al., 2013).

In contrast, longer $1 \mathrm{~h}$ photoillumination produced sleep only during the dark phase (Fig. 2) and not during the light phase (Fig. 2), which may seem paradoxical. However, since there is relatively little sleep early in the dark phase, even a small amount of sleep induced through inhibition of Hcrt neural activity can result in a significant change in sleep amounts. Conversely, a $1 \mathrm{~h}$ photoillumination during the light phase fails to produce significant effects due to a "ceiling effect" since this is the major sleep period for mice and the time of day when Hcrt/orexin levels are minimal.

\section{Novel observations in Orexin/Ataxin-3 (ATAX) mice}

Because loss of Hcrt neurons in ATAX mice provides face validity with NT1, this model has been used for pharmacological studies (Xie et al., 2008; Hara et al., 2009; Okuro et al., 2010; Black et al., 2013, 2014, 2017b; Sagawa et al., 2016). The original description of this strain described behavioral arrests resembling cataplexy, disrupted sleep architecture, reduced wake bout durations in 
both light and dark phases, more REM sleep in the dark phase due to longer bouts, reduced LMA, and late-onset obesity despite lower food consumption than nontransgenic littermates (Hara et al., 2001). We confirmed virtually all of these features, including cataplexy (Fig. 5Aiv), reduced wake bout durations (Fig. 5Ai) and activity (Fig. 4Aii) during the dark phase, and disrupted sleep architecture (increased numbers of wake and NREM bouts during the dark phase and across the $24 \mathrm{~h}$ period; Fig. $5 \mathrm{~A}$ ), and increased weight gain relative to WT mice (Fig. $4 B$, inset) despite reduced FI (Fig. 4Aii). In addition, we find that ATAX mice have reduced RER during the dark phase. These results confirm that ATAX mice replicate the metabolic and arousal state abnormalities characteristic of NT1.

The present study also extends characterization of sleep-wake abnormalities in ATAX mice. Although these mice entrained normally to an LD cycle, they had a longer circadian period than WT mice or either orexin/Arch subgroup (Fig. 4E), ATAX mice drifted very little from $24 \mathrm{~h}(\mathrm{tau}=23.90 \pm 0.06 \mathrm{~h})$. Cataplexy in ATAX mice was greatly exacerbated in the CCA (Fig. 5Biv vs Fig. 5Aiv). On the other hand, the EEG spectra (Fig. $5 C$ ) and homeostatic response to $4 \mathrm{~h} \mathrm{SD}$ (Fig. 6A) were unchanged in ATAX mice relative to WT. Sleep homeostasis has been reported as normal (Dantz et al., 1994) or enhanced in narcoleptic patients (Tafti et al., 1992) and other narcoleptic models (Mochizuki et al., 2004; Vassalli and Franken, 2017). ATAX mice had faster REM sleep onset in 4 of the 5 nap opportunities during the mMSLT than the other strains tested, further validating this strain as a model of NT1.

\section{aHE Orexin/Arch mice: a possible model of narcolepsy Type 2 (NT2)?}

ATAX and orexin-tTA;TetO-DTA mice (Tabuchi et al., 2014) are accepted NT1 models because of their Hcrt/orexin neuron loss, cataplexy, disrupted sleep architecture, and metabolic abnormalities. Short latency REM onsets during the mMSLT resemble the sleep onset REM periods that are diagnostic of human NT1, which is also characterized by low CSF Hcrt-1/orexin-A levels. In contrast, Type 2 narcolepsy (NT2) shares some NT1 features, such as EDS and sleep onset REM periods, but without cataplexy and usually with normal CSF Hcrt-1/orexin-A levels (Scammell, 2015; Black et al., 2017a; Bassetti et al., 2019; Szabo et al., 2019). A subset (10\%-20\%) of NT2 patients may progress to NT1 over their lifetime, but the large majority of NT2 patients will not; nonprogressing NT2 patients are thought to have a distinct disorder that may involve dysfunction of the Hcrt system without the massive cell loss found in NT1 (Bassetti et al., 2019; Szabo et al., 2019).

The abnormal Hcrt neuron properties in aHE mice (Fig. 3) raise the question whether this strain could be a useful NT2 model. aHE mice exhibited obesity as well as lower RER values during the light phase but greater RER during the dark phase than age-matched WT mice (Fig. 4Aii). Low RER values during the light phase have also been reported in aged Hcrt/orexin ligand KO mice (Ramanathan and Siegel, 2014). The increased RER in the dark phase was present in both orexin/Arch subgroups, but ATAX mice had the reverse pattern. Activity measured in the CLAMS system was elevated in the dark phase in both orexin/ Arch strains (Fig. 4Aii) but, when provided access to a running wheel, aHE mice ran less than any other strain (Fig. 4E; Table 2). Although many factors could contribute to reduced activity, other mouse models with compromised Hcrt signaling are less willing to work for a positive reward (McGregor et al., 2011), the emotional valence that is associated with running activity.
aHE mice also had more REM bouts than other strains across the $24 \mathrm{~h}$ period (Fig. 5Aiii) and a shorter REM latency under baseline conditions (Fig. 6B) but not on the mMSLT (Fig. 6E). The aHE phenotype was distinct from ATAX mice as aHE mice showed $24 \mathrm{~h} \mathrm{FI}$ (Fig. 4Aii), circadian periodicity (Fig. 4E), and REM latency on mMSLT that was equivalent to WT mice and, like NT2 patients, they lacked cataplexy (Fig. 5Aiv). Of all the strains examined, aHE mice consistently showed the most abnormal EEG spectra, with increased beta power in wake and further trends for altered spectra bands in NREM and REM (Fig. 5C).

For aHE mice to be considered as a model for NT2, key features, such as the absence of cataplexy, reduced sleep latency, increased sleep fragmentation, EDS, SOREM, and normal levels of hypocretin/orexin expression, should be present. We found that aHE mice lack cataplexy (Fig. 5Aiv), have increased REM sleep (Fig. 5Aiii), reduced sleep latency under basal conditions (Fig. $6 B$ ), and normal orexin cell counts. However, aHE mice did not present sleep-wake fragmentation, nor did we observe EDS or SOREM during the mMSLT evaluation. Thus, although aHE mice share some, but not all, of the characteristics of NT2, they may be the best available model to study this disorder.

\section{Limitations of viral versus transgenic approaches to optogenetics}

Previous optogenetic studies have either used viral-mediated or transgenic approaches to introduce light-sensitive proteins into the Hcrt neurons. Appropriate expression of light-sensitive proteins via viral delivery depends upon the accuracy of stereotaxic injections, efficacy of viral penetration into the relevant neuronal population, and subsequent gene transcription and translation of the protein. The efficiency of this approach has been greatly aided by Cre-dependent viruses and transgenic mouse strains that express Cre recombinase. In contrast, transgenic mice can provide stable expression, which may enable more reproducible results, although ectopic expression can be problematic. The present study demonstrates another limitation of transgenic lines: the phenotype observed may be due to disruption of normal cellular function due to the level of opsin expression. In the present study, the phenotypic characterization of orexin/Arch mice at the cellular and organismal levels documented as many differences between aLE and aHE mice as between either subgroup and WT mice, underscoring the need for thorough characterization of transgenic models. Such extensive phenotyping may avoid inappropriate attribution of a gain or loss of function to an intervention, such as optogenetic stimulation, which may indeed be due to transgene expression-dependent modification of cellular properties and concomitant downstream effects.

\section{References}

Adamantidis AR, Zhang F, Aravanis AM, Deisseroth K, de Lecea L (2007) Neural substrates of awakening probed with optogenetic control of hypocretin neurons. Nature 450:420-424.

Apergis-Schoute J, Iordanidou P, Faure C, Jego S, Schöne C, Aitta-Aho T, Adamantidis A, Burdakov D (2015) Optogenetic evidence for inhibitory signaling from orexin to $\mathrm{MCH}$ neurons via local microcircuits. J Neurosci 35:5435-5441.

Bassetti CL, Adamantidis A, Burdakov D, Han F, Gay S, Kallweit U, Khatami R, Koning F, Kornum BR, Lammers GJ, Liblau RS, Luppi PH, Mayer G, Pollmächer T, Sakurai T, Sallusto F, Scammell TE, Tafti M, Dauvilliers Y (2019) Narcolepsy: clinical spectrum, aetiopathophysiology, diagnosis and treatment. Nat Rev Neurol 15:519-539.

Black SW, Morairty SR, Fisher SP, Chen TM, Warrier DR, Kilduff TS (2013) Almorexant promotes sleep and exacerbates cataplexy in a murine model of narcolepsy. Sleep 36:325-336. 
Black SW, Morairty SR, Chen TM, Leung AK, Wisor JP, Yamanaka A, Kilduff TS (2014) GABAB agonism promotes sleep and reduces cataplexy in murine narcolepsy. J Neurosci 34:6485-6494.

Black SW, Yamanaka A, Kilduff TS (2017a) Challenges in the development of therapeutics for narcolepsy. Prog Neurobiol 152:89-113.

Black SW, Schwartz MD, Chen TM, Hoener MC, Kilduff TS (2017b) Trace amine-associated receptor 1 agonists as narcolepsy therapeutics. Biol Psychiatry 82:623-633.

Carter ME, Adamantidis A, Ohtsu H, Deisseroth K, de Lecea L (2009) Sleep homeostasis modulates hypocretin-mediated sleep-to-wake transitions. J Neurosci 29:10939-10949.

Carter ME, Brill J, Bonnavion P, Huguenard JR, Huerta R, de Lecea L (2012) Mechanism for hypocretin-mediated sleep-to-wake transitions. Proc Natl Acad Sci U S A 109:E2635-E2644.

Chow BY, Han X, Dobry AS, Qian X, Chuong AS, Li M, Henninger MA, Belfort GM, Lin Y, Monahan PE, Boyden ES (2010) High-performance genetically targetable optical neural silencing by light-driven proton pumps. Nature 463:98-102.

Dantz B, Edgar DM, Dement WC (1994) Circadian rhythms in narcolepsy: studies on a 90 minute day. Electroencephalogr Clin Neurophysiol 90:24-35.

Date Y, Ueta Y, Yamashita H, Yamaguchi H, Matsukura S, Kangawa K, Sakurai T, Yanagisawa M, Nakazato M (1999) Orexins, orexigenic hypothalamic peptides, interact with autonomic, neuroendocrine and neuroregulatory systems. Proc Natl Acad Sci U S A 96:748-753.

Franklin KB, Paxinos G (2008) The mouse brain in stereotaxic coordinates, Ed 3. Amsterdam: Elsevier.

Funato H, Tsai AL, Willie JT, Kisanuki Y, Williams SC, Sakurai T, Yanagisawa M (2009) Enhanced orexin receptor-2 signaling prevents diet-induced obesity and improves leptin sensitivity. Cell Metab 9:64-76.

Han X, Boyden ES (2007) Multiple-color optical activation, silencing, and desynchronization of neural activity, with single spike temporal resolution. PLoS One 2:e299.

Han X, Chow BY, Zhou H, Klapoetke NC, Chuong A, Rajimehr R, Yang A, Baratta MV, Winkle J, Desimone R, Boyden ES (2011) A high-light sensitivity optical neural silencer: development and application to optogenetic control of non-human primate cortex. Front Syst Neurosci $5: 18$.

Hara J, Beuckmann CT, Nambu T, Willie JT, Chemelli RM, Sinton CM, Sugiyama F, Yagami K, Goto K, Yanagisawa M, Sakurai T (2001) Genetic ablation of orexin neurons in mice results in narcolepsy, hypophagia, and obesity. Neuron 30:345-354.

Hara J, Gerashchenko D, Wisor JP, Sakurai T, Xie X, Kilduff TS (2009) Thyrotropin-releasing hormone increases behavioral arousal through modulation of hypocretin/orexin neurons. J Neurosci 29:3705-3714.

Inutsuka A, Inui A, Tabuchi S, Tsunematsu T, Lazarus M, Yamanaka A (2014) Concurrent and robust regulation of feeding behaviors and metabolism by orexin neurons. Neuropharmacology 85:451-460.

Irmak SO, de Lecea L (2014) Basal forebrain cholinergic modulation of sleep transitions. Sleep 37:1941-1951.

Jego S, Glasgow SD, Herrera CG, Ekstrand M, Reed SJ, Boyce R, Friedman J, Burdakov D, Adamantidis AR (2013) Optogenetic identification of a rapid eye movement sleep modulatory circuit in the hypothalamus. Nat Neurosci 16:1637-1643.

Konadhode RR, Pelluru D, Blanco-Centurion C, Zayachkivsky A, Liu M, Uhde T, Glen WB Jr, van den Pol AN, Mulholland PJ, Shiromani PJ (2013) Optogenetic stimulation of MCH neurons increases sleep. J Neurosci 33:10257-10263.

Li SB, Giardino WJ, de Lecea L (2017) Hypocretins and arousal. Curr Top Behav Neurosci 33:93-104.

Mahler SV, Moorman DE, Smith RJ, James MH, Aston-Jones G (2014) Motivational activation: a unifying hypothesis of orexin/hypocretin function. Nat Neurosci 17:1298-1303.

McGregor R, Wu MF, Barber G, Ramanathan L, Siegel JM (2011) Highly specific role of hypocretin (orexin) neurons: differential activation as a function of diurnal phase, operant reinforcement versus operant avoidance and light level. J Neurosci 31:15455-15467.

Mochizuki T, Crocker A, McCormack S, Yanagisawa M, Sakurai T, Scammell TE (2004) Behavioral state instability in orexin knock-out mice. J Neurosci 24:6291-6300.

Morairty SR, Dittrich L, Pasumarthi RK, Valladao D, Heiss JE, Gerashchenko D, Kilduff TS (2013) A role for cortical nNOS/NK1 neurons in coupling homeostatic sleep drive to EEG slow wave activity. Proc Natl Acad Sci U S A 110:20272-20277.

Oishi Y, Williams RH, Agostinelli L, Arrigoni E, Fuller PM, Mochizuki T, Saper CB, Scammell TE (2013) Role of the medial prefrontal cortex in cataplexy. J Neurosci 33:9743-9751.

Okuro M, Fujiki N, Kotorii N, Ishimaru Y, Sokoloff P, Nishino S (2010) Effects of paraxanthine and caffeine on sleep, locomotor activity, and body temperature in orexin/ataxin-3 transgenic narcoleptic mice. Sleep 33:930-942.

Peyron C, Tighe DK, van den Pol AN, de Lecea L, Heller HC, Sutcliffe JG, Kilduff TS (1998) Neurons containing hypocretin (orexin) project to multiple neuronal systems. J Neurosci 18:9996-10015.

Ramanathan L, Siegel JM (2014) Gender differences between hypocretin/ orexin knockout and wild type mice: age, body weight, body composition, metabolic markers, leptin and insulin resistance. J Neurochem 131:615-624.

Sagawa Y, Sato M, Sakai N, Chikahisa S, Chiba S, Maruyama T, Yamamoto J, Nishino S (2016) Wake-promoting effects of ONO-4127Na, a prostaglandin DP1 receptor antagonist, in hypocretin/orexin deficient narcoleptic mice. Neuropharmacology 110:268-276.

Sakurai T (2007) The neural circuit of orexin (hypocretin): maintaining sleep and wakefulness. Nat Rev Neurosci 8:171-181.

Sakurai T, Moriguchi T, Furuya K, Kajiwara N, Nakamura T, Yanagisawa M, Goto K (1999) Structure and function of human prepro-orexin gene. J Biol Chem 274:17771-17776.

Sakurai T, Nagata R, Yamanaka A, Kawamura H, Tsujino N, Muraki Y, Kageyama H, Kunita S, Takahashi S, Goto K, Koyama Y, Shioda S, Yanagisawa M (2005) Input of orexin/hypocretin neurons revealed by a genetically encoded tracer in mice. Neuron 46:297-308.

Scammell TE (2015) Narcolepsy. N Engl J Med 373:2654-2662.

Scammell TE, Willie JT, Guilleminault C, Siegel JM (2009) A consensus definition of cataplexy in mouse models of narcolepsy. Sleep 32:111-116.

Schöne C, Cao ZF, Apergis-Schoute J, Adamantidis A, Sakurai T, Burdakov D (2012) Optogenetic probing of fast glutamatergic transmission from hypocretin/orexin to histamine neurons in situ. J Neurosci 32:1243712443.

Schöne C, Apergis-Schoute J, Sakurai T, Adamantidis A, Burdakov D (2014) Coreleased orexin and glutamate evoke nonredundant spike outputs and computations in histamine neurons. Cell Rep 7:697-704.

Schuld A, Hebebrand J, Geller F, Pollmächer T (2000) Increased body-mass index in patients with narcolepsy. Lancet 355:1274-1275.

Schuld A, Beitinger PA, Dalal M, Geller F, Wetter TC, Albert ED, Hebebrand J, Pollmächer T (2002) Increased body mass index (BMI) in male narcoleptic patients, but not in HLA-DR2-positive healthy male volunteers. Sleep Med 3:335-339.

Schwartz MD, Black SW, Fisher SP, Palmerston JB, Morairty SR, Hoener MC, Kilduff TS (2017) Trace amine-associated receptor 1 regulates wakefulness and EEG spectral composition. Neuropsychopharmacology 42: $1305-1314$.

Suzuki A, Sinton CM, Greene RW, Yanagisawa M (2013) Behavioral and biochemical dissociation of arousal and homeostatic sleep need influenced by prior wakeful experience in mice. Proc Natl Acad Sci U S A 110:10288-10293.

Szabo ST, Thorpy MJ, Mayer G, Peever JH, Kilduff TS (2019) Neurobiological and immunogenetic aspects of narcolepsy: implications for pharmacotherapy. Sleep Med Rev 43:23-36.

Tabuchi S, Tsunematsu T, Black SW, Tominaga M, Maruyama M, Takagi K, Minokoshi Y, Sakurai T, Kilduff TS, Yamanaka A (2014) Conditional ablation of orexin/hypocretin neurons: a new mouse model for the study of narcolepsy and orexin system function. J Neurosci 34:6495-6509.

Tafti M, Rondouin G, Besset A, Billiard M (1992) Sleep deprivation in narcoleptic subjects: effect on sleep stages and EEG power density. Electroencephalogr Clin Neurophysiol 83:339-349.

Tsujino N, Yamanaka A, Ichiki K, Muraki Y, Kilduff TS, Yagami K, Takahashi S, Goto K, Sakurai T (2005) Cholecystokinin activates orexin/hypocretin neurons through the cholecystokinin A receptor. J Neurosci 25:74597469 .

Tsunematsu T, Kilduff TS, Boyden ES, Takahashi S, Tominaga M, Yamanaka A (2011) Acute optogenetic silencing of orexin/hypocretin neurons induces slow-wave sleep in mice. J Neurosci 31:10529-10539.

Tsunematsu T, Tabuchi S, Tanaka KF, Boyden ES, Tominaga M, Yamanaka A (2013) Long-lasting silencing of orexin/hypocretin neurons using ar- 
chaerhodopsin induces slow-wave sleep in mice. Behav Brain Res 255: $64-74$.

Tsunematsu T, Ueno T, Tabuchi S, Inutsuka A, Tanaka KF, Hasuwa H, Kilduff TS, Terao A, Yamanaka A (2014) Optogenetic manipulation of activity and temporally controlled cell specific ablation reveal a role for MCH neurons in sleep-wake regulation. J Neurosci 34:6896-6909.

Van Dort CJ, Zachs DP, Kenny JD, Zheng S, Goldblum RR, Gelwan NA, Ramos DM, Nolan MA, Wang K, Weng FJ, Lin Y, Wilson MA, Brown EN (2015) Optogenetic activation of cholinergic neurons in the PPT or LDT induces REM sleep. Proc Natl Acad Sci U S A 112:584-589.

Vassalli A, Franken P (2017) Hypocretin (orexin) is critical in sustaining theta/gamma-rich waking behaviors that drive sleep need. Proc Natl Acad Sci U S A 114:E5464-E5473.

Veasey SC, Yeou-Jey H, Thayer P, Fenik P (2004) Murine multiple sleep latency test: phenotyping sleep propensity in mice. Sleep 27:388-393.

Xie X, Wisor JP, Hara J, Crowder TL, LeWinter R, Khroyan TV, Yamanaka A, Diano S, Horvath TL, Sakurai T, Toll L, Kilduff TS (2008) Hypocretin/ orexin and nociceptin/orphanin FQ coordinately regulate analgesia in a mouse model of stress-induced analgesia. J Clin Invest 118:2471-2481.

Xu M, Chung S, Zhang S, Zhong P, Ma C, Chang WC, Weissbourd B, Sakai N,
Luo L, Nishino S, Dan Y (2015) Basal forebrain circuit for sleep-wake control. Nat Neurosci 18:1641-1647.

Yamanaka A, Tsujino N, Funahashi H, Honda K, Guan JL, Wang QP, Tominaga M, Goto K, Shioda S, Sakurai T (2002) Orexins activate histaminergic neurons via the orexin 2 receptor. Biochem Biophys Res Commun 290:1237-1245.

Yamanaka A, Muraki Y, Tsujino N, Goto K, Sakurai T (2003a) Regulation of orexin neurons by the monoaminergic and cholinergic systems. Biochem Biophys Res Commun 303:120-129.

Yamanaka A, Beuckmann CT, Willie JT, Hara J, Tsujino N, Mieda M, Tominaga M, Yagami Ki, Sugiyama F, Goto K, Yanagisawa M, Sakurai T (2003b) Hypothalamic orexin neurons regulate arousal according to energy balance in mice. Neuron 38:701-713.

Yoshida K, McCormack S, España RA, Crocker A, Scammell TE (2006) Afferents to the orexin neurons of the rat brain. J Comp Neurol 494: $845-861$.

Zhang F, Wang LP, Brauner M, Liewald JF, Kay K, Watzke N, Wood PG, Bamberg E, Nagel G, Gottschalk A, Deisseroth K (2007) Multimodal fast optical interrogation of neural circuitry. Nature 446:633-639. 